Sādhanā, Vol. 19, Part 5, October 1994, pp. 671-703. (C) Printed in India.

\title{
Buoyant plane plumes from heated horizontal confined wires and cylinders
}

\author{
GUY LAURIAT $^{1}$ and GILLES DESRAYAUD ${ }^{2}$ \\ ${ }^{1}$ Laboratoire de Thermique, CNAM, 292 rue Saint-Martin, 75141 Paris, \\ Cedex 03, France \\ ${ }^{2}$ INSSET, Université de Picardie, 48 rue Raspail, BP 422, 02109 \\ Saint-Quentin, Cedex, France
}

\begin{abstract}
Two-dimensional computations are reported for timedependent laminar buoyancy-induced flows above a horizontal heated source immersed in an air-filled vessel. Two kinds of heated source were considered: a line heat source, modelled as a heat source term in the energy equation, and a heat-flux cylinder of small diameter. First, comparisons are presented for the results obtained for these two heated sources. Rather large discrepencies between the velocity fields appeared in the conduction regime due to the weak plume motion, while close agreements were found in the boundary layer regime. Nevertheless, same types of bifurcations occur with almost identical frequencies, whatever the Rayleigh number. It is concluded that for dimensions of the enclosures, which largely compared with the cylinder radius, the heat source term model is a promising way to study the behaviour of unsteady plumes owing to its simplicity, flexibility, and low computational costs. Second, transitions to unsteady flows were studied through direct flow simulations for various depths of immersion of a line heat source in the central vertical plane of a vessel. Different routes to chaos were shown to occur according to the aspect ratio of the vessel and the depth of immersion of the line source. Three distinct regimes were detected with different underlying physical mechanisms called natural swaying motion, penetrative convection and Rayleigh-Benard-like convection. The first bifurcations associated with these regimes are supercritical Hopf bifurcation, pitchfork bifurcation and subcritical Hopf bifurcation. Comparisons with experimental results of confined buoyant plumes above heated wires show very good agreement with laminar frequency correlations.
\end{abstract}

Keywords. Buoyant plane plumes; line heat source; heat-flux cylinder; numerical plume simulations; route to chaos.

\section{Introduction}

Free laminar convection from a horizontal line heat source has received increasing attention during past decades due to the importance of this process, which occurs in 
many engineering and natural systems of practical interest such as electronic packages, geophysical systems and nuclear reactors. Although a number of analytical and experimental investigations have been devoted to freely rising plumes, many experimental works have been conducted in confined spaces either to minimize environmental effects or to study the behaviour of confined plumes. On the other hand, a literature survey reveals that very little numerical work has been devoted to confined buoyant plumes; the increase in complexity caused by the strong interaction between the plume and its surroundings due to confinement and thermal boundary conditions, in addition to its oscillatory behaviour makes it difficult to perform a parametric study of the different routes to chaos.

Classical self-similar solutions were widely used in early theoretical studies (Fujii 1963; Gebhart et al 1970; Fujii et al 1973; see also Gebhart et al 1988, chap. 3). However, the coupled differential equations for laminar plane plumes were analytically solved in closed form only for specific values of the Prandtl numbers, i.e. $\mathrm{Pr}=5 / 9$ (Crane 1959) and $\operatorname{Pr}=2$ (Fujii 1963; Gebhart et al 1970; Fujii et al 1973). The selfsimilar analyses were extended to second-order (Hieber \& Nash 1975) and third-order boundary layer theories (Mörwald et al 1986). Recently, a correlation for the centreline temperature has been developed by Lin \& Cheng (1992). This correlation equation is valid over the entire buoyancy regime and for any Prandtl number.

During the same period, many experiments were conducted in air, water, silicone fluid and spindle oil (Brodowicz \& Kierkus 1966; Lyakhov 1970; Schorr \& Gebhart 1970; Gebhart et al 1970; Fujii et al 1973; Nawoj \& Hickman 1977). To explain the discrepancies between analytical and experimental results for centreline temperature and velocity distributions, the concept of virtual line source was introduced by Forstrom \& Sparrow (1967), Lyakhov (1970), Hieber \& Nash (1975) and Yosinobu et al (1979). In this way, it was attempted to explain the divergence of the plume flow from the ideal due to the finite diameter of the wire used to simulate the line source. However, this approach raised some controversies (Schorr \& Gebhart 1970; Fujii et al 1973; Nawoj \& Hickman 1977) and did not account for the entire 15-20\% difference between experimental and theoretical results; neither end-conduction effects nor decrease of the plume velocity near both ends of the line source could account for them. Yosinobu et al (1979) attributed these differences to the heat losses below the wire caused by fluid entrainment. Lyakhov (1970) found only a weak difference on bounding the space below the line source with an impermeable insulating plate; this is more consistent with the boundary layer theory, which did. not take into account convective motion below the source. Mörwald et al (1986) drew attention to the fact that such discrepancies did not appear from measurements in water. They argued that the Grashof numbers based on diameter and heat rate input of the heat sources were much larger than one for water only, while Gr were of the order of one for air, spindle oil and silicone fluids. This could be a possible explanation regarding the origin of the discrepancies, since at low Grashof number, the flow field around the wire contradicts the basic assumptions of the boundary-layer theory, i.e. slender plumes.

Forstrom \& Sparrow (1967) were the first to observe a naturally swaying motion of a plume which was studied a short time later by Schorr \& Gebhart (1970) through flow visualizations. Following the observations of regular swaying plumes, linear stability analysis of freely rising plumes based on the quasi-parallel theory was performed by Pera \& Gebhart (1971) and later by Wakitani \& Yosinobu (1984), but they failed to find a critical Grashof number. Haaland \& Sparrow (1973) and Hieber \& 
Nash (1975) obtained lower branches of neutral curves (and then critical Grashof numbers) by taking some non-parallel and higher-order effects of the base flow into account in the linear stability analysis. This indicates that two-dimensional disturbances are amplified selectively (see also Gebhart et al 1988, chap. 11). More recently, Wakitani (1985) using a non-parallel theory (the WKB method) confirmed their results except for the amplification rate of disturbances within unstable regions. The experimental results of Bill \& Gebhart (1975) for a plane plume subjected to naturally occurring disturbances and those of Pera \& Gebhart (1971), Yosinobu et al (1979) and Wakitani \& Yosinobu (1984) for controlled disturbances confirmed the prediction of the linear stability analysis over a wide range of Rayleigh numbers. It has been demonstrated that sufficiently high frequency disturbances are stable, as they are convected downstream.

The transition from a laminar to a turbulent state in a freely rising plume was experimentally investigated by Forstrom \& Sparrow (1967), Bill \& Gebhart (1975), Yosinobu et al (1979) and Noto (1989), and in stable-thermally stratified fluid inside a large enclosure by Noto 't al (1982). Forstrom \& Sparrow (1967) and Bill \& Gebhart (1975) determined the beginning of the transition to be at Rayleigh numbers $\mathrm{Ra}_{x} \cong 3.6 \times 10^{8}$ and $\mathrm{Ra}_{x} \cong 8 \times 10^{8}$ respectively, while for a thermal plume in air Noto (1989) obtained $7 \times 10^{7} \leqslant \mathrm{Ra}_{x} \leqslant 1.4 \times 10^{8}$ from spectral analysis of thermocouple signals and change of the slope of the midplane temperature (the Rayleigh number being based on the vertical distance along the plume, and on the heat rate input). Noto (1989) related the large discrepancies found in the critical Rayleigh number to the different methods used in its determination.

By a different formulation of the onset of instability in plume flow, the buckling theory, Kimura \& Bejan (1983) and Yang (1992) demonstrated that at the laminarturbulent transition the buoyant plume assumes a sinusoidal shape which is characterized by a non-axisymmetric deformation; the sinuous mode having the highest growth rate. Their theoretical arguments are strongly supported by experimental evidence (Kimura \& Bejan 1983).

Igarashi \& Kada (1977) carried out experiments in confined spaces to investigate the natural convective oscillatory motion of air caused by a heated wire placed concentrically along the axis of a horizontal isothermal cylinder. Different diameters of wires (from $0.29 \mathrm{~mm}$ to $1.0 \mathrm{~mm}$ ) and outer cylinders (from $100 \mathrm{~mm}$ to $194.5 \mathrm{~mm}$ ) were used. The same results were obtained regardless of the diameter of the wire. By performing a dimensional analysis to determine the relationship between the frequency of the oscillatory flow, the thermal and geometrical conditions, and the fluid properties, they concluded that the frequency only depends on the diameter of the outer cylinder and on the heat input, and not on the diameter of the wire or its temperature. Thus, the wall temperature of the outer cylinder was chosen as the reference temperature for the fluid properties rather than the film temperature. Furthermore, a modified Rayleigh number based on the heat flux input and on the radius of the outer cylinder was introduced. The relationship between the non-dimensional frequency and the modified Rayleigh number was found to be $f=C \mathrm{Ra}^{0.4}$. Igarashi \& Kada (1977) also determined the initiation conditions at the onset of oscillations. For a given outer cylinder diameter, they found that the swaying motion did not appear when the heat flux input exceeded some critical values but rather occurred when the temperature difference between the wire and the cooled cylinder exceeded a critical threshold. They suggested that the onset of oscillatory motion is governed by the fluid state close to the wire, whereas the frequency of the oscillations is governed by 
the outer boundary conditions far from the wire. Their work was extended by Igarashi (1978) in the case of a line heat source placed concentrically along the axis of a horizontal rectangular chamber. The same conclusions as for the cylinder configuration were arrived at and a correlation having the same power value was established. However, due to the additional geometrical parameter, i.e. the height of the cross-section of the chamber, the $C$-coefficient has to be a function of the aspect ratio of the chamber if the Rayleigh number is based on the width. Three distinct values of $C$ were found corresponding to the three distinct steady flow patterns, giving way to different frequency regimes. Igarashi (1978) also demonstrated the existence of a relationship between the frequency and the middle plane vertical velocity far above the wire and suggested that the plume oscillations are related to the flow circulation rate.

Eichhorn \& Vedhanayagam (1982) determined analytically a power value of 0.3 which correlated their experimental results for a water turbulent plume within $\pm 10 \%$. A $1 / 3$ power value was also found both by Urakawa et al (1983) for the swaying frequency of a spindle oil plume with a free surface and by Wakitani \& Yosinobu (1984) for a laminar air plume. Noto \& Matsumoto (1986) and Noto (1989) found a swaying frequency of the thermal air plume proportional to the 0.4 or $1 / 3$ power of the heat rate depending on whether the plume reaches the ceiling of the enclosure in a laminar or a turbulent state respectively.

A meandering motion was also noted in experiments on confined plumes by Fujii et al (1973, 1982), Eichhorn \& Vedhanayagan (1982) and Urakawa et al (1983); experiments performed in large parallelepipedic enclosures not only showed that the plume sways in a plane perpendicular to the wire but that it can also meander in the direction of the wire, i.e. across the span of the plume. However it should be noted that the underlying physical mechanisms are not yet clarified. Eichhorn et al (1974) and Incropera \& Yaghoubi (1980) in experimental studies of immersed isothermal cylinders also observed "transition from 2 to 3-dimensional instability, with increased axial twisting or billowing". This transition was attributed to fluid entrainment effects by Incropera \& Yaghoubi (1980). When a meandering motion exists, these two periodic motions (meandering and swaying) are not independent of each other: the swaying motion is stable only when the meandering waveforms along the heater are stable. This happens when the heater length equals integral multiples of a half wavelength, the meandering wave being a fairly precise sine curve (Eichhorn \& Vedhanayagam 1982; Urakawa et al 1983). The liquid surface height (depth of immersion), linearly related to the meandering wavelength, and the length of the heater are the main parameters controlling the meandering waveform. Pera \& Gebhart (1971), Nawoj \& Hickman (1977), Yosinobu et al (1979), Noto et al (1982) and Noto (1989) did not find any meandering motion in their experiments. Noto related the meandering motion to the width of the enclosure apparently without experimental proof: meandering waveforms would appear only for small widths of enclosures. It is worth noting that meandering motions were mainly observed in free-surface fluid experiments with heated wires or cylinders. Fujii et al (1973) carried out experiments in air, water and spindle oil and meandering waves only occurred in the case of liquids. Only Bill \& Gebhart (1975) mentioned oscillations along the wire span for air plume by interferogram visualizations of plume cross-section.

Numerical and experimental work for heated wires located just below a free surface was recently conducted by Maquet et al (1992) and Rozé et al (1993). Maquet et al (1992) carried out numerical experiments for a square pool with a free surface and 
differentially heated sidewalls in which a wire of constant temperature was located below the horizontal free surface. The buoyancy and surface tension mechanisms were incorporated into their formulation. From steady state calculations, they concluded that, even if the temperature differences are small, the free surface deformation is not small with respect to the depth of immersion of the wire. For a $20 \mu$ m-diameter wire, Rozé et al (1993) experimentally observed a steady surface deformation taking the shape of a trough when the wire was very close to the free surface and the shape of a crest when the depth of immersion was of the order of $1 \mathrm{~mm}$. These two effects were related to surface tension and buoyancy mechanisms respectively. By increasing the wire temperature, an oscillatory motion around the wire arose simultaneously with free-surface propagating waves, these two phenomena having the same frequency. While this bifurcation was recognized as supercritical Hopf bifurcation, they remarked that some liquids (such as silicone oil) developed such oscillatory motions while others (such as water) were not. Moreover, secondary instabilities were not observed beyond the Hopf bifurcation.

Fujii et al (1973) were the first to observe a deflection of the plume towards one of the walls when the temperatures of the vertical walls were slightly different. This deflection inhibits the swaying motion. Later on, some experimental studies were devoted to plume interactions (Pera \& Gebhart 1975; Incropera \& Yaghoubi 1980) or to the influence of solid or liquid interfaces on heat transfer of buoyant plumes (Reimann 1974). Jaluria (1982) investigated the interaction between a plume and a vertical unheated surface and showed that the basic mechanism of the deflection process is the limitation of the flow which supplies the fluid entrained downstream by the plume. Incropera \& Yaghoubi (1980) observed various modes of plume interactions from an array of horizontal cylinders: interaction might occur at the air-water surface, before reaching the interface by forming well-ordered ascending and descending flows, or resulting in a highly disordered flow with many recirculating regions of varying sizes. However, the type of plume interaction was shown not to have any influence on the general nature of the temperature distribution.

Although considerable analytical and numerical efforts have been devoted to the study of self-similar solutions for freely-rising plumes, the thermal plumes were confined inside vessels in many experimental investigations. In these cases, the ascending fluid is cooled at a horizontal solid or free surface, inducing a recirculating flow along the sidewalls and an entrainment of underlying fluid. Despite the contributions of the above quoted experimental studies, much remains to be learnt concerning the interaction of the plume motion with its surroundings, in particular with the top surface. To the authors' knowledge, only a few attempts have been made to numerically simulate thermal plumes inside rectangular vessels. Amongst them are the recent studies of Peyret (1990) for double diffusive convection, Xia et al (1990, 1991) for an externally heated enclosure containing a local heat source of finite size, and Maquet et al (1992) who took into account Marangoni effects. Although most plumes occurring in the environment are turbulent, it is felt that deeper investigation of the behaviour of laminar plumes is justified; since many numerical codes developed to study laminar flows are now used for direct simulations of chaotic or weakly turbulent flows, it is important to ascertain how these laminar models behave. Of course, direct simulations are very time-consuming, even when running on powerful vector computers. Therefore, simplifications of the complexity in the governing equations or in the numerical procedure are not only helpful but necessary. Moreover, the restriction to two-dimensional flow simulations precludes the study of three- 
dimensional effects along the line source. Such a limitation is imposed by the computers presently available. However, a two-dimensional model is of interest to provide insight into the occurrence of the swaying motion and also into the transition from periodicity to chaos in considerable detail. Indeed, almost all the experiments highlight a swaying motion of the plume in the cross-section of the wire while very few detect a meandering motion in the spanwise direction of the wire.

Our ultimate purpose being to develop a reliable numerical scheme which would be fast enough to investigate laminar-turbulent transition plume flows and $3 \mathrm{D}$ parametric study, it is shown in the present paper that the wire may be modelled as a local source term in the energy equation. Other strategies have been employed such as boundary-fitted coordinates (Himasekar \& Bau 1988) or the finite elements method for cylindrical configurations, but at the expense of additional complexities in the numerical treatment of the governing equations. Hybrid coordinate systems have also been used in the vicinity of the cylinder (Fujii et al 1982; Farouk \& Shayer 1985). Such a procedure allows better control of grid locations but has the disadvantage of the presence of an overlapping zone between the two different grid systems and requires the introduction of somewhat arbitrary conditions where the meshes meet. Another advantage of the local heat source term strategy is its inherent flexibility since it permits the heat source to be moved easily. Also, thermal interactions between two or more line sources inside vessels could readily be studied.

The formulations and the numerical methods used in the present study of dynamical and thermal hehaviour of a plume above a heat-flux wire immersed in a rectangular vessel are described in the two following sections $(\$ \S 2$ and 3 ). The first part of $\S 4$ deals with comparisons between results obtained when modelling the wire as a source term in the energy equation or when considering it as a uniform heat-flux cylinder of small diameter. The second part focusses on the different routes to chaos found in square and rectangular vessels according to the depth of immersion of a wire. Almost all these results were presented in four published papers (Lauriat \& Desrayaud 1990; Desrayaud \& Lauriat 1991, pp. 609-21, 1993; Deschamps \& Desrayaud 1994).

\section{Mathematical formulation}

\subsection{Buoyant plume around a heated cylinder}

Consider a two-dimensional fluid-filled vessel of width $L$ and depth $H$ enclosed by adiabatic vertical walls and isothermally cooled horizontal surfaces at $T_{a}$. A cylinder of diameter $d^{\prime}$ centred at the point $\left(x_{s}^{\prime}, y_{s}^{\prime}\right)$ generating a heat flux $Q$ per unit length $(\mathrm{W} / \mathrm{m})$ is immersed in the central vertical plane of the vessel (figure 1a). The $y^{\prime}$-axis points upwards. The third dimension of the vessel is taken to be sufficiently large so that a two-dimensional approximation of the flow could be assumed valid.

For a Boussinesq fluid, the conservation equations for mass, momentum and energy are reduced in the dimensionless form to

$$
\begin{gathered}
\nabla \cdot \mathbf{V}=0 \\
\frac{1}{\operatorname{Pr}\left[\frac{\partial \mathbf{V}}{\partial t}+(\mathbf{V} . \nabla) \mathbf{V}\right]}=-\nabla p+\nabla^{2} \mathbf{V}+\operatorname{Ra} \theta \mathbf{k} \\
\frac{\partial \theta}{\partial t}+(\mathbf{V} . \nabla) \theta=\nabla^{2} \theta
\end{gathered}
$$



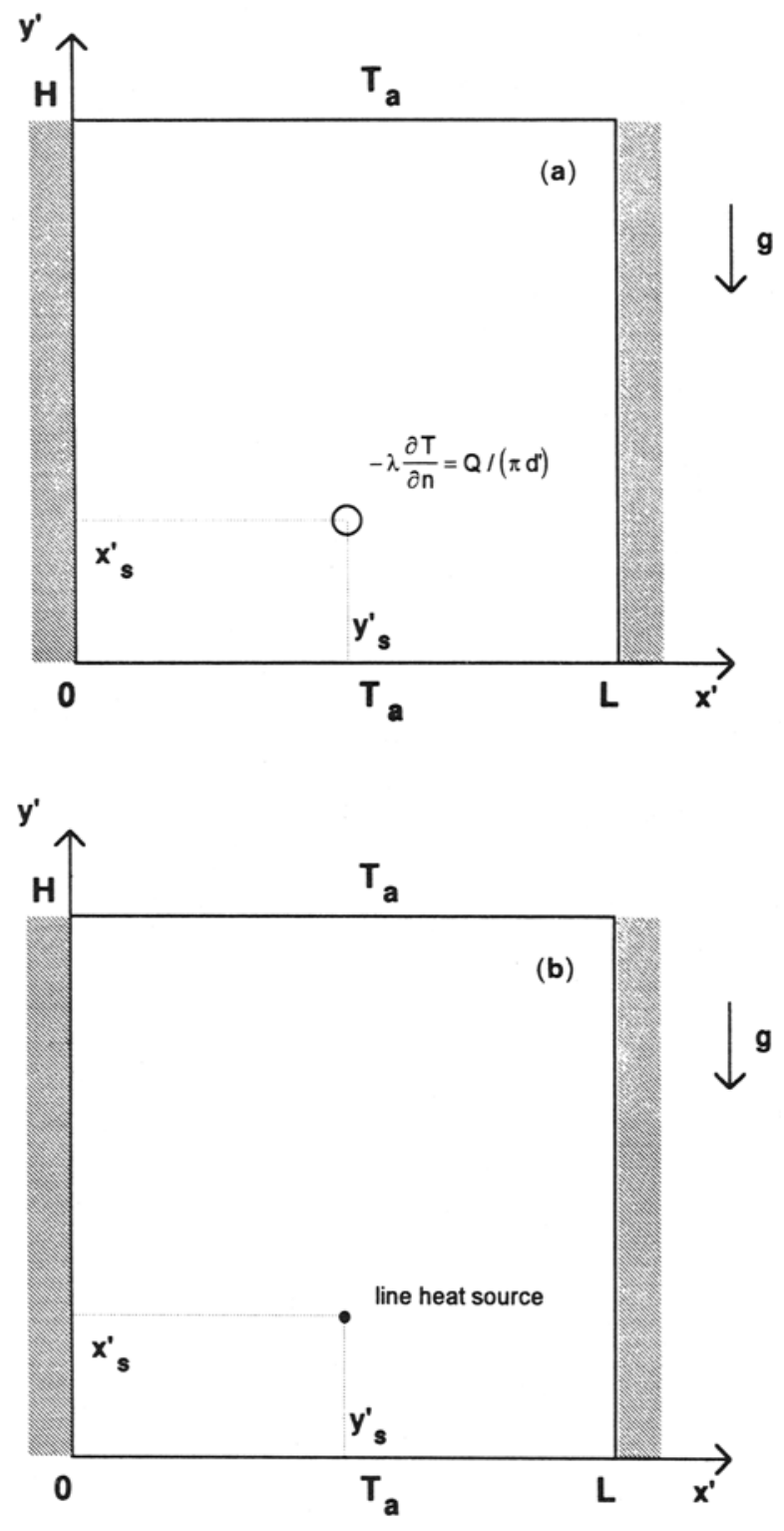

Figure 1. (a) Coordinate system for a cylinder, $A=1, H_{s}=0.75$; (b) coordinate system for a line heat source, $A=1, H_{s}=0.75$.

where the components of the velocity vector $\mathrm{V}$ are $(U, V)$ in the $(x, y)$ directions. The Prandtl and Rayleigh numbers are defined as $\operatorname{Pr}=v_{0} / a_{0}$ and $\mathrm{Ra}=g \beta Q L^{3} / \lambda_{0} v_{0} a_{0}$ respectively.

The dimensionless form of (1)-(3) has been obtained by scaling lengths, time and temperature difference $\left(T-T_{a}\right)$ by $L, L^{2} / a_{0}$ and $Q / \lambda_{0}$ respectively. Here $a_{0}$ and $v_{0}$ are the thermal and viscous diffusivities respectively, $\lambda_{0}$ the thermal conductivity, $\rho_{0}$ the fluid density, $\beta$ the coefficient of volume expansion and $g$ the gravitational acceleration; the subscript 0 denotes thermophysical properties at the ambient temperature $T_{a}$, chosen as the reference temperature.

As a result, the relevant hydrodynamic and thermal boundary conditions can be 
written in the following dimensionless form

$$
\begin{array}{rlrl}
\mathrm{V} & =0, & & \text { at all solid boundaries, } \\
\frac{\partial \theta}{\partial x} & =0, & \text { at } \quad x=0,1, \\
\theta & =0, \quad \text { at } \quad y=0, A,
\end{array}
$$

where $A=H / L$ is the aspect ratio of the cross section of the vessel.

$$
\frac{\partial \theta}{\partial \mathbf{n}}=-1 /(\pi d), \quad \text { at the cylinder boundary, }
$$

n being the dimensionless outward unit vector normal to the cylinder surface and $d$ the dimensionless diameter of the cylinder.

\subsection{Buoyant plume around line heat source}

We now consider two-dimensional convection induced by a line heat source immersed at the dimensionless point $\left(x_{s}, y_{s}\right)$ in a Boussinesq fluid. The line source has been modelled as a local source term in the energy equation. This requires that the diameter of a real heat source be much smaller than the dimensions of the vessel. It is convenient to introduce the dimensionless stream function $\psi$ and the vorticity $\Omega$ such that

$$
\mathbf{V}=(U, V)=(-\partial \psi / \partial y, \partial \psi / \partial x) \text { and } \mathbf{\Omega}=-\nabla^{2} \psi
$$

Hence, the governing equations for the vorticity and the temperature are

$$
\begin{aligned}
\frac{1}{\operatorname{Pr}}\left(\frac{\partial \Omega}{\partial t}+\nabla \cdot(\Omega \mathbf{V})\right) & =\nabla^{2} \Omega-\operatorname{Ra} \frac{\partial \theta}{\partial x} \\
\frac{\partial \theta}{\partial t}+\nabla \cdot(\theta \cdot \mathbf{V}) & =\nabla^{2} \theta+\varepsilon .
\end{aligned}
$$

The boundary conditions $(4 a-c)$ are also imposed at the walls of the vessel.

When modelling a pipe as a line source, Beck et al (1988) used Green's functions and developed a transient solution to describe heat conduction around a single buried steam pipe inside a semi-infinite medium. The basic idea of modelling the wire as a source term in the energy equation is then to cast the source term $\varepsilon$ as follows

$$
\varepsilon=\delta\left(x_{s}-x\right) \delta\left(y_{s}-y\right),
$$

where $\delta(z)$ is the Dirac delta function. The integral of $\delta\left(x_{s}-x\right) \delta\left(y_{s}-y\right)$ over $x$ and $y$ equals unit, if it includes $\left(x_{s}, y_{s}\right)$; otherwise it is zero. Peyret (1990) used a similar technique but with an exponential decay of the intensity of the source term so that it could be represented accurately with spectral decomposition.

At steady state, the energy dissipated by the line heat source is lost at the boundaries. This requirement of equality leads to the non-dimensional steady state condition,

$$
\int_{\mathbb{D}} \delta\left(x_{s}-x\right) \delta\left(y_{s}-y\right) \mathrm{d} S=1=-\int_{\Gamma} \frac{\partial \theta}{\partial n} \mathrm{~d} \Gamma,
$$

where $\mathbb{D}$ is the problem domain and $\Gamma$ the boundary. 
For adiabatic vertical walls, the heat flux through any horizontal plane $y$, defined as

$$
\phi(y)=\int_{0}^{1}\left(-\frac{\partial \theta}{\partial y}+V \theta\right) d x,
$$

must satisfy the following conditions:

$$
\phi\left(y^{-}\right)+\phi\left(y^{+}\right)=1, \quad \forall y^{-}<y_{s} \text { and } \forall y^{+}>y_{s} .
$$

Finally, the depth of immersion $H_{s}=A-y_{s}$ is defined as the vertical distance of the line source or the centre of the cylinder with respect to the top surface of the vessel.

\section{Numerical procedure}

\subsection{Numerical method for the finite-diameter cylinder}

A finite element method was used for solving the governing PDEs in the primitive variable formulation, (1) - (3), with the boundary conditions given by (4a-d). While finite elements are CPU-time consuming, modelling a cylinder inside a rectangular vessel is straightforward. This is why a commercial fluid dynamics analysis package (FIDAP 1991) was employed.

A major difficulty in the application of the Galerkin finite element method for the incompressible Navier-Stokes equations is the elimination of spurious pressure modes. As a remedy for the checker-board pressure modes, a mixed-interpolation method was used, i.e. the interpolation of the pressure was a polynomial at least one order lower than that for the velocity. However, this method does not always provide accurate pressure fields, especially with bilinear interpolation for velocity and piecewise constant pressure (i.e., the $Q_{1} / P_{0}$ finite element). The elimination of the pressure via a penalty function approach not only reduces the size of the linear system but eliminates the spurious pressures. The combination of the mixed interpolation method and the penalty formulation with exact (consistent) integration for the penalty term is one of the basic algorithms for the treatment of the velocity-pressure coupling of incompressible flows in FIDAP (1991). It is worth noting that the exact integration is equivalent to the reduced integration technique for the $Q_{1} / P_{0}$ finite element and results in the same penalty matrix (Engelman et al 1982).

For the present computations, the four-node quadrilateral finite element was used for velocity and temperature variables with piecewise constant pressure and penalty function approximation. Integrals were evaluated exactly using one point Gaussian quadrature. At every selected Rayleigh number, the successive substitution method was employed for the first three iterations when solving the system of nonlinear equations, while the Newton-Raphson method was chosen after the third iteration. With these combinations, solutions converged smoothly to a $0.1 \%$ convergence criterion of the relative velocity and residuals within six to seven iterations for all steps. The selected penalty constant was $10^{-8}$ for all cases and Rayleigh numbers.

Finite element meshes were built over a Cartesian $33 \times 33$ grid and cylindrical mesh refinement was only used near the cylinder (figure 2); mesh refinement is needed in the vicinity of the cylinder when decreasing its size in order to maintain the accuracy of the results, the four-node quadrilateral element being straight-sided. The numbers of four-node quadrilateral elements and nodal points used for different diameters of 


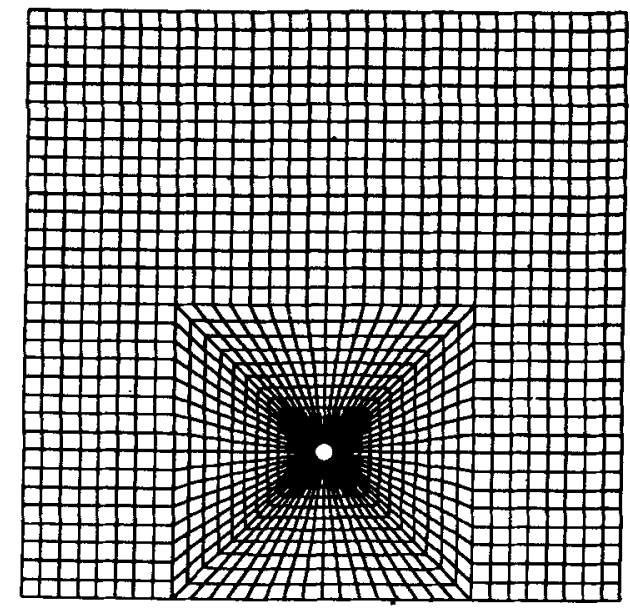

Figure 2. Finite element grid.

cylinder inside a square vessel are reported in table 1 . Thus, 1152 four-node elements were used for the $1 / 10$ diameter cylinder while 1664 elements were used for $d=1 / 100$. Another consequence of mesh refinement was that, after the three successive substitution iterations, four Newton-Raphson iterations were needed to reach the convergence criterion for $d=1 / 100$ instead of 3 for greater diameters. Thus, the elapsed CPU time greatly increased for very small diameters.

A nine-node quadrilateral Lagrangian finite element for the velocity and temperature associated with linear discontinuous pressures, the $Q_{2} / P_{1}$ finite element, was also tested (Deschamps \& Saiac 1992). Some numerical tests of FIDAP have also been performed for some classical benchmarks by Sohn (1988) showing the efficiency of the $Q_{2} / P_{1}$ finite element for the treatment of velocity-pressure coupling of incompressible fluid flow. This finite element has the major advantage of not presenting checker-board pressure modes. The penalty function method was preferred instead of the integrated method (for which the pressure variables contribute an additional degree of freedom and are built on linear continuous interpolation functions) because the memory storage required for fine meshes was found prohibitive and significantly

Table 1. Number of four-node elements according to cylinder diameter in a square vessel $\left(H_{\mathrm{s}}=0.75, \mathrm{Ra}=10^{5}\right)$. Maxima are grid-values and the subscript 1 is for the point $M_{1}(0.5,3 \mathrm{~A} / 4)$.

\begin{tabular}{|c|c|c|c|c|c|c|c|c|}
\hline \multirow[b]{2}{*}{ Diameter } & \multicolumn{2}{|c|}{ Number of } & \multicolumn{2}{|c|}{$\begin{array}{l}\text { Iterative } \\
\text { methods }\end{array}$} & \multirow[b]{2}{*}{$\psi_{\max }$} & \multirow[b]{2}{*}{$V_{\max }$} & \multirow[b]{2}{*}{$U_{\max }$} & \multirow[b]{2}{*}{$\theta_{1}$} \\
\hline & $\begin{array}{l}\text { Nodal } \\
\text { points }\end{array}$ & Elements & SS & NR & & & & \\
\hline $1 / 10$ & 1248 & 1152 & 3 & 3 & $5 \cdot 34$ & $42 \cdot 04$ & $22 \cdot 86$ & 0.133 \\
\hline $1 / 20$ & 1376 & 1280 & 3 & 3 & 5.65 & $45 \cdot 30$ & $23 \cdot 80$ & 0.137 \\
\hline $1 / 50$ & 1504 & 1408 & 3 & 3 & 5.83 & 46.98 & $24 \cdot 36$ & 0.139 \\
\hline $1 / 70$ & 1632 & 1536 & 3 & 3 & 5.87 & 47.88 & 24.99 & 0.140 \\
\hline $1 / 100$ & 1760 & 1664 & 3 & 4 & 5.90 & $48 \cdot 25$ & $24 \cdot 58$ & $0 \cdot 140$ \\
\hline
\end{tabular}

SS: successive substitution method; NR: Newton-Raphson method 
Table 2. Comparisons of some characteristic values for two different finite element discretizations and two Rayleigh numbers in a square vessel.

\begin{tabular}{|c|c|c|c|c|c|c|c|c|c|}
\hline \multirow[b]{2}{*}{$\mathrm{Ra}$} & \multirow[b]{2}{*}{$\begin{array}{l}\text { Element } \\
\text { type }\end{array}$} & \multicolumn{2}{|c|}{ Number of } & \multicolumn{2}{|c|}{$\begin{array}{l}\text { Iterative } \\
\text { methods }\end{array}$} & \multirow[b]{2}{*}{$\psi_{\max }$} & \multirow[b]{2}{*}{$V_{\max }$} & \multirow[b]{2}{*}{$U_{\max }$} & \multirow[b]{2}{*}{$\theta_{1}$} \\
\hline & & $\begin{array}{l}\text { Nodal } \\
\text { points }\end{array}$ & Elements & SS & NR & & & & \\
\hline \multirow{2}{*}{$10^{5}$} & 4-node & 1632 & 1536 & 3 & 3 & $5 \cdot 87$ & $47 \cdot 88$ & $24 \cdot 49$ & 0.140 \\
\hline & 9-node & 1632 & 384 & 3 & 3 & 5.83 & $47 \cdot 79$ & $24 \cdot 37$ & $0 \cdot 139$ \\
\hline \multirow{2}{*}{$10^{7}$} & 4-node & 1632 & 1536 & & 7 & $46 \cdot 7$ & $446 \cdot 6$ & $327 \cdot 6$ & 0.057 \\
\hline & 9-node & 1632 & 384 & & 7 & $46 \cdot 0$ & $445 \cdot 2$ & 318.9 & 0.056 \\
\hline
\end{tabular}

$d=1 / 70, H_{\mathrm{s}}=0.75\left(\mathrm{at} \mathrm{Ra}=10^{7}\right.$ a guess solution was used).

SS: successive substitution method; NR: Newton-Raphson method

Subscript 1 is for the point $M_{1}(0 \cdot 5,3 A / 4)$

increased the CPU time. Table 2 presents some local values in a square vessel for two different Rayleigh numbers. In order to avoid dramatic increases of the CPU time, about the same number of nodal points were used in both cases. Nevertheless, the CPU time was greater for the nine-node element, especially for the highest Rayleigh number (about 25\% more). Consequently, all the runs were performed with the four-node element, the nine-node element showing unexpectedly slight accuracy improvement (Huyakorn et al 1978).

For transient problems, the time integration was performed by the combination of two accurate second-order techniques: the implicit trapezoid rule for the velocity and an explicit Adams-Bashforth formula for the pressure. If the solution at each time step is reasonably close to the solution at the previous time step, a one-step Newton-Raphson method can be used to solve the nonlinear system of algebraic equations. Typically, a value of $\Delta t=0.003$ was used at $\mathrm{Ra}=10^{7}$ in a square vessel for a cylinder having a diameter of $1 / 70$. All the finite elements computations were performed on an superscalar workstation.

\subsection{Numerical method for the line heat source}

In the case of the local source term strategy, the vorticity and energy equations (5)-(7) were solved in transient form and the time integration was performed using an alternating directional implicit (ADI) splitting scheme. The vorticity equation was discretized by employing second-order central differences based on Taylor series expansions for all spatial derivatives, including the convective terms. The nodal points were located on a standard mesh. For the energy equation, a control-volume formulation with staggered grids and central differencing was retained in order to improve the overall energy balance. One layer of grid points outside each boundary was included to facilitate the application of the boundary conditions using quadratic extrapolations. For both vorticity and energy equations, the Thomas algorithm was employed to solve the tridiagonal systems of algebraic equations. On the other hand, finite difference equations for the stream function equation were solved by a direct method which uses a block-cyclic reduction process (Golub \& Meurant 1983). From 
the solution of the stream function equation, the wall vorticities were updated using an accurate second-order formulation based on Jensen's estimate in conjunction with Briley's formula (Roache 1982, p. 142). On account of the expected flow structure, uniform grids were used for all the computations discussed in the present paper. However, it should be noted that the main limitation of the direct method which was used is that the number of mesh points in one of the directions has to be chosen as a power of 2 . Double precision computations were found necessary to compute accurately the threshold values and the characteristics of the oscillatory motions.

The computer code was validated with the steady and oscillatory solutions of a benchmark problem of natural convection of low-Prandtl fluids in differentially heated shallow cavities (Desrayaud et al 1990). Very good agreement was found both in the overall (general trends of unsteady flows) and pointwise comparisons (stream function and velocity maxima) with other computational methods: finite volume, finite element and spectral (Roux et al 1990).

Several common indicators of dynamics were employed for tracking convergence to an asymptotic state. Global indicators were calculated by using the discrete $L_{1}$ or $L_{2}$ norms on the flow data or on the flow change data. These are:

- the relative $L_{1}$ norm of the stream function change per time step

$$
\|\psi\|_{1}=\Sigma\left|\psi^{n+1}-\psi^{n}\right| / \Sigma\left|\psi^{n+1}\right|
$$

- the total kinetic energy per surface unit: $K E=(1 / 2 \mathbb{D})\|V\|_{d, 2}^{2}$;

- the heat flux $\phi$ through the top wall. In some cases, upper half heat fluxes, right $\phi_{r}$ and left $\phi_{l}$, have been used.

- the $L_{2}$ norm of the temperature per surface unit: $P E=(1 / \mathbb{D})\|\theta\|_{d, 2}^{2}$.

Since small-scale flow features can be masked by global indicators, a series of local variables, such as wall vorticity, velocity components or temperature were also recorded.

For the problem reported here, a number of tests were performed with various grid sizes and time steps to ensure accuracy and stability and to avoid spurious aperiodic flows (Desrayaud \& Lauriat 1991). It was observed that the vorticity value in the vertical central plane was the most sensitive indicator, not only when symmetrybreaking transition occurred but also when the flow underwent a Hopf bifurcation.

Most of the computations were performed on an IBM $3090600 / \mathrm{VF}$ vector computer. For typical cases, the vectorized performance (ratio of scalar to vectorial CPU time) was only slightly greater than two. The reason for this is mainly the difficulty of vectorizing the bloc-cyclic reduction solver. When using an ADI scheme to solve the Poisson equation of the stream function, the vectorized performance was over three. However, it is preferable not to introduce an internal iterative procedure when accurately transient motions are followed.

\subsection{Convergence history}

Calculations for the line heat source solution were done on three different regular grid structures in square vessels. Grid tests were conducted to ensure that the results were independent of both the grid density and the size of the source. Table 3 shows partial results from the tests for Rayleigh numbers below the critical value $\left(R_{c} \cong 3.1\right.$ $10^{7}$ ). The local and overall data from these calculations differed by less than one 
Table 3. Comparison of some characteristic values for different Rayleigh numbers in a square vessel and for a depth of immersion $H_{\mathrm{s}}=0.75$ in the case of a line heat source. Subscript 1 is for the point $M_{1}(0 \cdot 5,3 A / 4)$.

\begin{tabular}{lccccccc}
\hline Ra & Grid & $\phi$ & $\theta_{s}$ & $\psi_{\max }$ & $V_{\max }$ & $U_{\max }$ & $\theta_{1}$ \\
\hline \multirow{2}{*}{$10^{5}$} & $33 \times 33$ & 0.688 & 0.281 & 6.13 & 49.70 & 24.80 & 0.140 \\
& $65 \times 65$ & 0.687 & 0.376 & 6.14 & 50.52 & 25.01 & 0.141 \\
& $129 \times 129$ & 0.687 & 0.480 & 6.14 & 50.73 & 25.09 & 0.141 \\
$10^{6}$ & $33 \times 33$ & 0.813 & 0.125 & 18.52 & 157.9 & 97.41 & 0.085 \\
& $65 \times 65$ & 0.812 & 0.250 & 18.53 & 162.0 & 101.3 & 0.086 \\
& $129 \times 129$ & 0.811 & 0.343 & 18.56 & 163.0 & 101.9 & 0.086 \\
$10^{7}$ & $33 \times 33$ & 0.886 & 0.102 & 46.05 & 418.9 & 290.3 & 0.052 \\
& $65 \times 65$ & 0.881 & 0.157 & 46.45 & 438.9 & 316.3 & 0.055 \\
& $129 \times 129$ & 0.879 & 0.231 & 46.60 & 444.5 & 322.3 & 0.055 \\
\hline
\end{tabular}

percent for the two finest meshes. Note that maxima are grid-point values. For all the cases, the data were mostly grid-dependent around the heat source; since the heat is introduced into one control volume only, the temperature and flowfield in the immediate vicinity of the heat source cannot be grid-independent. The source temperature must tend to infinity as the area of the control volume tends towards zero. The decrease in the source temperature $\left(\theta_{s}\right)$ when the Rayleigh number is increased is due to the choice of the dimensionless variables. It is worth noticing that the results are satisfactory even for the coarsest mesh (within $5 \%$ ), showing that stationary flow could be modelled using quite coarse meshes. Furthermore, two different regular grid structures were used to test the grid independence in a rectangular vessel of aspect ratio 2 (table 4$)$. For these cases $\left(H_{s}=1\right)$, the onset of periodic motion arises at low Rayleigh numbers and coarse grids thus give accurate results (within $1 \%$ ) for stationary motions.

Extensive grid testing was also conducted for periodic motions. Test runs were made in square vessel at high Rayleigh numbers (table 5) and in a rectangular vessel at low Rayleigh numbers for various depths of immersion (table 6). It is seen that a strong dependency on the critical Rayleigh number is only found at high Rayleigh numbers, while a coarse grid gives accurate threshold and periodic motion at low Rayleigh numbers in the rectangular vessel whatever the depth of immersion. It demonstrated that, even if a weak dependence on mesh size is found for stationary

Table 4. Comparison of some characteristic values for symmetric and asymmetric flows in a rectangular vessel $\left(A=2, H_{s}=1\right)$. Subscript 2 is for the point $M_{2}(0 \cdot 25$, $3 A / 4)$.

\begin{tabular}{rccccccc}
\hline Ra & Grid & $\phi$ & $\psi_{\max }$ & $V_{1}$ & $\theta_{1}$ & $U_{2}$ & $V_{2}$ \\
\hline $10^{3}$ & $33 \times 49$ & 0.509 & 0.14 & 0.00 & 0.265 & -0.152 & 0.258 \\
& $65 \times 97$ & 0.509 & 0.14 & 0.00 & 0.265 & -0.152 & 0.259 \\
$5 \times 10^{3}$ & $33 \times 49$ & 0.619 & 2.22 & 2.62 & 0.305 & -2.21 & 2.91 \\
& $65 \times 97$ & 0.619 & 2.21 & 2.62 & 0.306 & -2.21 & 2.92 \\
\multirow{2}{*}{$10^{4}$} & $33 \times 49$ & 0.637 & 1.46 & 0.00 & 0.342 & -1.52 & 9.08 \\
& $65 \times 97$ & 0.637 & 1.47 & 0.00 & 0.343 & -1.53 & 9.13 \\
\hline
\end{tabular}


Table 5. Onset of periodic motion in a square cavity for a line heat source $\left(H_{\mathrm{s}}=0.75\right)$.

\begin{tabular}{lcc}
\hline Grid & $\begin{array}{c}\text { Ra } \\
\left(\times 10^{7}\right)\end{array}$ & Frequency \\
\hline $33 \times 33$ & 6.0 & $319.8 \pm 0.8$ \\
$65 \times 65$ & 3.2 & $308.2 \pm 0.6$ \\
$129 \times 129$ & 3.1 & $307.6 \pm 0.6$ \\
\hline
\end{tabular}

flows at high Rayleigh numbers (table 3), critical values and frequencies exhibit strong grid dependencies as soon as the flow undergoes Hopf bifurcations (table 5).

Furthermore, to ensure the independence of the periodic motion with regard to the time step, the solutions were computed for two different time steps, but only for the two coarsest meshes $(33 \times 33$ and $65 \times 65)$ in the square vessel. Frequencies were found to agree within one percent. Because hundreds of thousands of iterations are needed to reach established periodic motion through supercritical Hopf bifurcations, the $129 \times 129$ mesh flows were computed only once; computations for such a fine mesh are highly CPU-time consuming. Nevertheless, characteristics of the periodic motion are very similar to those found for the $65 \times 65$ mesh, which gives us confidence in these results.

However, care should be taken in choosing the time step, since spurious secondary bifurcations (quasi-periodic solutions) may appear during the time integration of periodic flows. Figure 3 presents the effects of too large a time-step $\left(\Delta t=10^{-4}\right.$ instead of $5 \times 10^{-5}$ ) on the transient. Starting from a steady motion at $\mathrm{Ra}=310^{7}$ in a square vessel, the flow undergoes a periodic state of frequency $f=294.6$ at a reduced time $t \cong 1 \cdot 7$. Therefore, using too large a time step yields at first an oscillatory solution with about the right frequency (table 5), but with a shortened transient motion, showing an amplification effect of the round-off errors. Beyond $t \cong 8$, this amplification effect produces a spurious aperiodic flow characterized by several low independent frequencies. This result shows the need to re-compute from time to time some oscillatory solutions using a sequence of time steps to check the validity of the oscillatory results.

Table 6. Frequencies for two uniform grids and for different depths of immersion in a rectangular vessel $(A=2)$, line heat source model.

\begin{tabular}{lcccc}
\hline $\begin{array}{l}\text { Depth of } \\
\text { immersion } \\
\left(H_{\mathrm{s}}\right)\end{array}$ & Grid & $\begin{array}{c}\text { Source } \\
\text { position }\end{array}$ & Ra & $\begin{array}{c}\text { Frequency } \\
( \pm 0 \cdot 12)\end{array}$ \\
\hline 1.50 & $33 \times 49$ & 17,13 & $2 \cdot 510^{4}$ & 2.07 \\
& $65 \times 97$ & 33,25 & $2.510^{4}$ & 2.07 \\
1.00 & $33 \times 49$ & 17,25 & $310^{4}$ & 5.61 \\
& $65 \times 97$ & 33,49 & $310^{4}$ & 5.62 \\
0.75 & $33 \times 49$ & 17,31 & $310^{5}$ & 15.17 \\
& $65 \times 97$ & 33,61 & $310^{5}$ & $15 \cdot 14$ \\
\hline
\end{tabular}




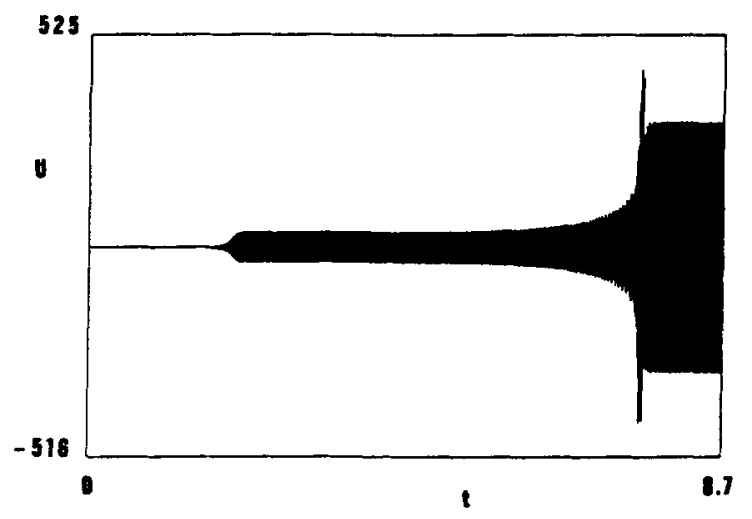

Figure 3. Time history from $\mathrm{Ra}=3 \times 10^{7}$ to $\mathrm{Ra}=3 \cdot 1 \times 10^{7}$ of the horizontal component of the velocity at point $(0 \cdot 5,2 A / 3)$ in a square vessel $(65 \times 65$ mesh $)$.

In transient motion leading to asymmetric steady flows, for depths of immersion greater than the width of the vessel ( $\$ 4.2 \mathrm{~d}$ ), figure 4 shows the general trends for $A=2$ and $H_{s}=1.75$. Starting from symmetric steady motion at $\mathrm{Ra}=10^{3}$ to $\mathrm{Ra}=6$ $10^{3}$, the global indicator on the flow change data decreases and becomes as small as $10^{-13}$. Then an opposite evolution occurs at $t \geqslant 8$, just after the break in the symmetry of the flow. At $t \geqslant 25$, the global indicator starts to decrease again. The time change is different for local indicators at the centre line of the vessel: the vorticity is equal to the zero machine for symmetric motion and increases continuously up to an asymptotic value as the time increases. Then, the flow reaches an asymmetric steady state motion (see figure 11 below).

Finally, figure 5 presents transient evolutions through a supercritical bifurcation like the ones arising when the depth of immersion is smaller than the width of the vessel $(\S \S 4 \cdot 2 b$ and $4 \cdot 2 c)$. Although the transition is easily detected in the case of subcritical Hopf bifurcations (since the oscillations arise with a finite amplitude), the transition from steady to oscillatory flows occurs through soft bifurcations such as the one displayed in figure 5. Such transitions can be the source of erroneous interpretations; the global indicators on the flow change data decrease to a level of the order of $10^{-10}$, while the local ones on the flow data stay at a relatively high level. In these cases, time integration must be pursued in order to show up a dramatic change. Thus, the usual indicators employed to stop the computations must only be used to scrutinize the transient behaviour of the flow in direct simulations of the route to chaos. Figures 4 and 5 show also that local indicators are more meaningful

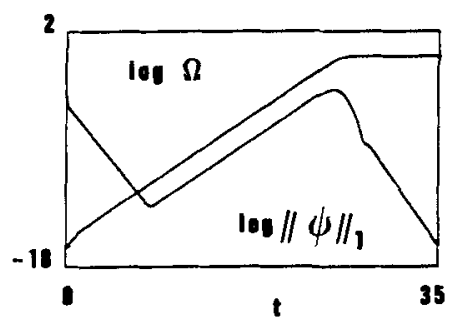

Figure 4. Time histories from $\mathrm{Ra}=10^{3}$ to $\mathrm{Ra}=6 \times$ $10^{3}$ of the logarithm of the vorticity at point $(0.5, A)$ and of the $L_{1}$ norm of stream function change in a rectangular vessel $\left(A=2, H_{s}=1 \cdot 75,33 \times 49 \mathrm{mesh}\right)$. 


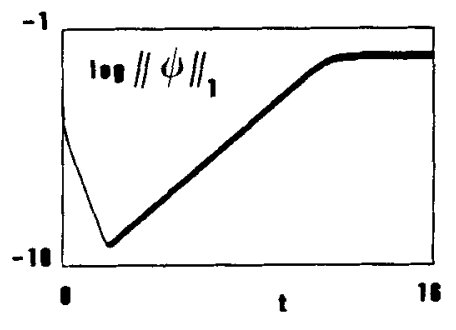

Figure 5. Time histories of the logarithm of relative $L_{1}$ norm of stream function change for supercritical Hopf bifurcation, $A=2, H_{\mathrm{s}}=$ $0.75, \mathrm{Ra}=3 \times 10^{5}$.

than global ones, although local indicators must be chosen carefully according to the characteristics of the flow.

All these tests were performed for a line heat source owing to the fairly low computational cost. The numerical solutions of the Navier-Stokes equations written in the stream function-vorticity formulation and discretized by finite volume methods are much less time-consuming than finite element methods for solving the $(u, v, p)$ equations, especially for transient motions.

\section{Resuits}

\subsection{Comparisons between the two approaches}

Figures $6(\mathrm{a}-\mathrm{c})$ show comparisons for steady state temperature and velocity profiles in a square vessel at $\mathrm{Ra}=10^{6}$. The depth of immersion is $H_{s}=0.75$ and the plots are for two cylinder sizes, of diameters $1 / 20$ and $1 / 70$, and for the heat source term modelling with a $65 \times 65$ mesh. The profiles for a $1 / 100$-diameter cylinder are not plotted here because no visible difference can be seen between them and those for the 1/70-diameter. The temperature profiles on the vertical centreplane (figure 6a) show that the temperatures tend towards those of a line heat source when the diameter of the cylinder decreases, although some differences exist below the heat source. The vertical velocity profile in the vertical middle plane $x=0.5$ is shown in figure $6 \mathrm{~b}$. As can be seen, a non-zero velocity exists at the line source when it is modelled as a heat source term. However, the agreement between the velocity profiles for the two approaches is rather good in the ranges $0 \leqslant y \leqslant 0.2$ and $0.4 \leqslant y \leqslant 1$ in the case of a $1 / 70$-diameter cylinder. Finally, the influence of the cylinder diameter on the vertical velocity profile in the horizontal plane $y=A-H_{3}$ (i.e. the plane passing through the centre of the cylinder) is displayed in figure $6 \mathrm{c}$. While two velocity maxima are seen on both sides of the cylinder, owing to the no-slip boundary conditions, and only one for the line heat source, the differences between the two models are small on the major part of the profiles. Obviously, the discrepancies in the vicinity of the cylinder decreases when decreasing the cylinder diameter.

Figure 7a shows the relative differences for the maximum of the stream function, $\psi_{\max }$, and for the heat flux through the top wall, $\phi$, as a function of the Rayleigh number when the cylinder diameter is increased from $d=1 / 100$ to $1 / 10$. The solid lines are for $\psi_{\max }$ while the dashed lines are for $\phi$. These are the 1,2 and $5 \%$ iso-difference curves, the reference value being the solution for a cylinder of $1 / 100$-diameter. For $\mathrm{Ra} \leqslant 10^{5}$, the motion is in the so-called conduction or transition regimes. More than $65 \%$ of the heat being transferred by conduction to the bottom 

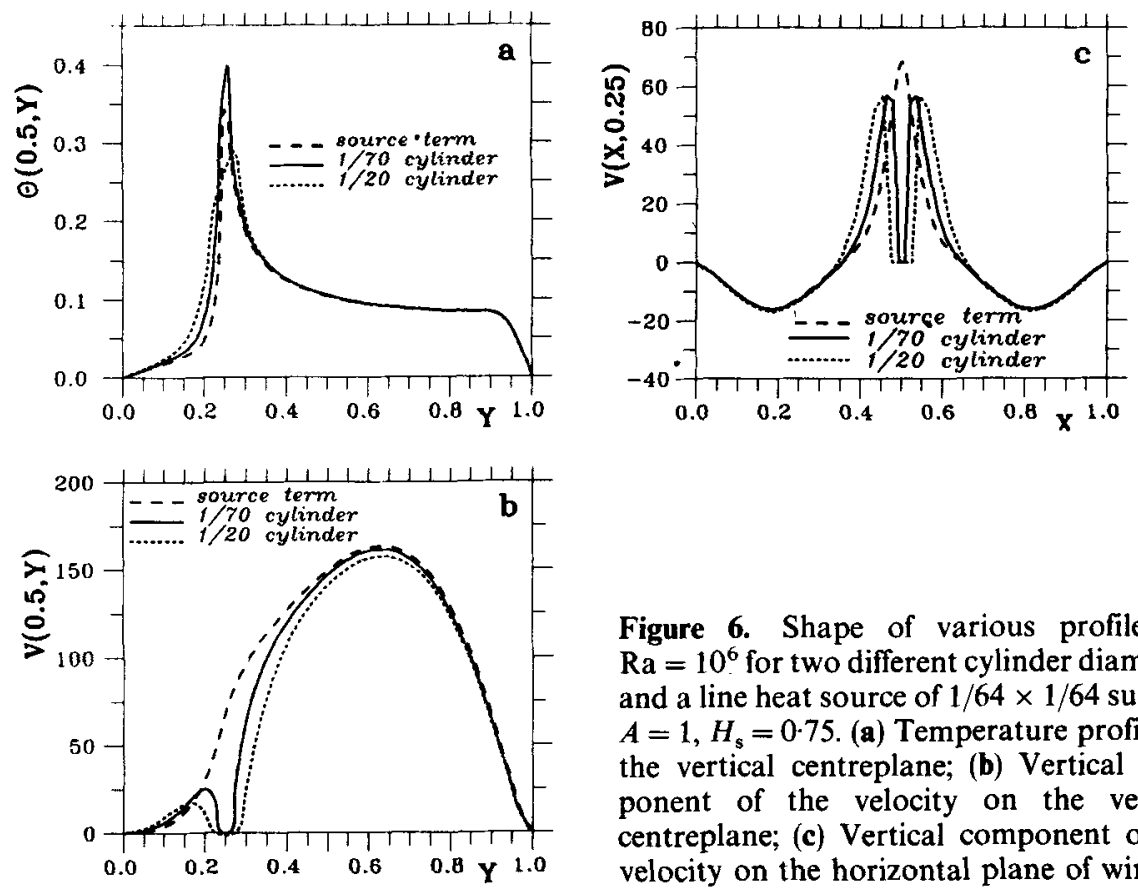

Figure 6. Shape of various profiles at $\mathrm{Ra}=10^{6}$ for two different cylinder diameters and a line heat source of $1 / 64 \times 1 / 64$ surface. $A=1, H_{\mathrm{s}}=0.75$. (a) Temperature profile on the vertical centreplane; (b) Vertical component of the velocity on the vertical centreplane; (c) Vertical component of the velocity on the horizontal plane of wire.

wall at $\mathrm{Ra}=10^{4}$, the temperature field is not very sensitive to the size of the cylinder. For $d=1 / 60$ and $1 / 20$, the relative differences in $\phi$ are then close to $1 \%$ and $5 \%$ respectively. On the contrary, larger discrepancies appear for the stream function because of the low velocity field. The cylinder is then an obstacle to the fluid motion, since the velocities are small: at $\mathrm{Ra}=10^{4}$, the differences in the stream function for $d=1 / 60$ is about $4 \%$ and reaches $17 \%$ for $d=1 / 20$. Thus, the flow is greatly affected

(a)

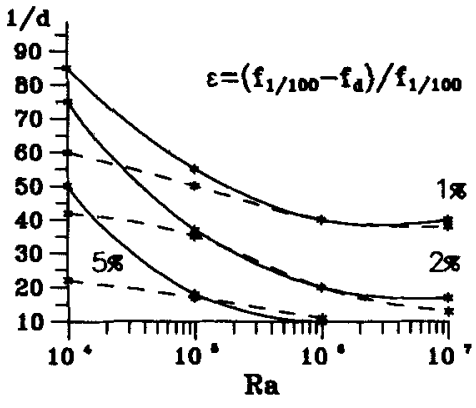

(b)

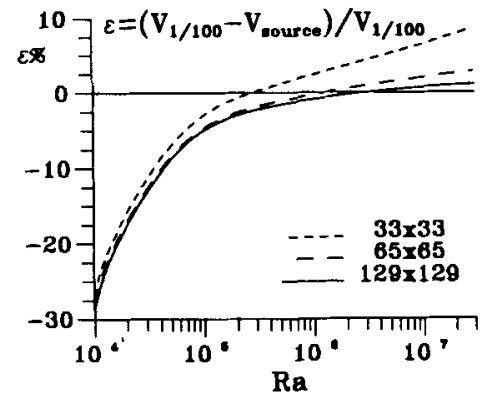

Figure 7. $A=1, H_{\mathrm{s}}=0.75$. (a) Curves of relative iso-difference for the cylinder model. Solid lines: stream function maximum, dashed lines: upper heat flux. (b) Curves of relative difference of $V_{\max }$ for different mesh sizes of the source term model. The reference is the $d=1 / 100$ cylinder. 
by the size of the cylinder in the conduction and transition regimes. On the other hand, the discrepancies are smaller in the boundary layer regime because heat is then mainly convected downstream by the plume. It appears that an asymptotic value of the diameter is reached for a given relative difference: $1 / 40$ for $1 \%$ and $1 / 20$ for $2 \%$. It occurs for a Rayleigh number close to $10^{6}$, i.e. at the beginning of the boundary layer regime.

Figure $7 \mathrm{~b}$ presents the evolution of the relative difference as a function of the Rayleigh number for the source term model and for the three meshes used: $33 \times 33$, $65 \times 65$ and $129 \times 129$. The comparisons are for the maximum of the vertical component of the velocity, which is always on the vertical centreplane of the vessel. According to $\mathrm{Ra}$, the location of the maximum velocity moves between $y=0.5$ and 0.7 . Here again, the reference value is the maximum vertical velocity in the vertical plane $y=0.5$ for a cylinder of diameter $d=1 / 10$ and the same conclusions as for figure $7 \mathrm{a}$ can be drawn. The largest discrepancies are about $30 \%$ for $\mathrm{Ra}=10^{4}$, whatever the mesh size. This relative difference decreases when the Rayleigh number increases and remains small for the two finest meshes, even at $\mathrm{Ra}=310^{7}$. For the coarsest mesh $(33 \times 33)$, it reaches $10 \%$. Nevertheless, it is worth noting that the curves for the three meshes exhibit the same trends.

The relative differences between the maxima of the vertical velocity component in the vertical middle plane and in the horizontal plane passing through the cylinder axis are reported in table 7 for various cylinder diameters and depths of immersion. On account of the symmetry of the flow, only one velocity maximum needs to be considered in the horizontal plane while two velocity maxima have to be considered in the vertical middle plane: one below the heated cylinder and one above, into the thermal plume. For all the data reported in table 7 , the reference values are the maximum velocities at the same planes for a cylinder with a diameter $d=1 / 100$. The relative differences between the velocity maxima in the horizontal plane are denoted $H$ and are reported in the left-hand columns of table 7 for the three depths of immersion investigated. The relative differences between the vertical velocity maxima are denoted $V A$ and $V B$, above and below the cylinder respectively. All the computations were carried out for $\mathrm{Ra}=10^{6}$. At this Rayleigh number, the plume flow is at the beginning of the boundary layer regime. For higher Rayleigh number, the differences are of the same order while they are greater for lower Rayleigh number because the motion is weaker.

As can be seen, the relative differences in the vertical centreplane (see $V A$ - and $V B$-columns) depend mainly on the diameter of the cylinder and are almost independent of the depth of immersion, except for $d=1 / 10$. These differences are a little less below the cylinder than above. However, the size of the cylinders greatly affects the motion, especially for $d \geqslant 1 / 30$. On the contrary, the relative differences in the horizontal plane of the cylinder are not only affected by the size of the cylinder but also by its location. Table 7 shows that these differences increase strongly with the depth of immersion.

The distances from the centre of the cylinder to the location of the three velocity maxima are also reported in table $7, \Delta x$ being on the horizontal plane and $\Delta y$ on the vertical centreplane. At constant depth of immersion, these values are almost constant provided $d<1 / 20$. For greater diameters, the maxima are slightly shifted away. This shows that the influence of the size of the cylinder on the flowfield is limited to the very near surroundings of the cylinder.

For periodic flows, computations were carried out for only one cylinder diameter, 
Table 7. Relative differences in percent between maximum of the vertical component of the velocity for various depths of immersion. $R a=10^{6} \mathrm{~A}=1$.

$\Delta x, \Delta y$ are the positions of the maxima from the centre of the cylinder

\begin{tabular}{|c|c|c|c|c|c|c|c|c|c|}
\hline \multirow[b]{2}{*}{$d$} & \multicolumn{3}{|c|}{$H_{\mathrm{s}}=0.25$} & \multicolumn{3}{|c|}{$H_{\mathrm{s}}=0.5$} & \multicolumn{3}{|c|}{$H_{\mathrm{s}}=0.75$} \\
\hline & $H(\%)$ & $V B(\%)$ & $V A(\%)$ & $H(\%)$ & $V B(\%)$ & $V A(\%)$ & $H(\%)$ & $V B(\%)$ & $V A(\%)$ \\
\hline $1 / 10$ & $22 \cdot 9$ & $28 \cdot 6$ & 57.4 & 37.9 & $14 \cdot 2$ & 53.8 & $62 \cdot 3$ & $13 \cdot 5$ & $46 \cdot 4$ \\
\hline${ }_{\Delta x}^{\Delta y}$ & 0.090 & 0.064 & 0.340 & 0.103 & 0.075 & 0.325 & 0.092 & 0.062 & 0.312 \\
\hline $\begin{array}{l}1 / 20 \\
\Delta y\end{array}$ & $9 \cdot 4$ & $\begin{array}{c}17.6 \\
0.41\end{array}$ & $\begin{array}{l}30 \cdot 5 \\
0 \cdot 314\end{array}$ & $22 \cdot 2$ & $\begin{array}{l}12 \cdot 9 \\
0 \cdot 049\end{array}$ & $\begin{array}{c}29.9 \\
0.299\end{array}$ & 37.8 & $\begin{array}{l}14.5 \\
0.035\end{array}$ & $\begin{array}{l}29 \cdot 1 \\
0 \cdot 286\end{array}$ \\
\hline$\Delta x$ & 0.063 & & & 0.075 & & & 0.061 & & \\
\hline $\begin{array}{l}1 / 30 \\
\Delta y\end{array}$ & 5.4 & $\begin{array}{c}11 \cdot 7 \\
0.041\end{array}$ & $\begin{array}{c}191 \\
0 \cdot 314\end{array}$ & $12 \cdot 6$ & $\begin{array}{l}9 \cdot 3 \\
0 \cdot 026\end{array}$ & $\begin{array}{l}18 \cdot 6 \\
0 \cdot 299\end{array}$ & $25 \cdot 6$ & $\begin{array}{c}11 \cdot 8 \\
0.025\end{array}$ & $\begin{array}{c}21.4 \\
0.275\end{array}$ \\
\hline$\Delta x$ & 0.063 & & & 0.075 & & & 0.061 & & \\
\hline $1 / 50$ & $2 \cdot 2$ & $\begin{array}{l}5 \cdot 7 \\
0.041\end{array}$ & $\begin{array}{l}8 \cdot 8 \\
0 \cdot 314\end{array}$ & $5 \cdot 2$ & $\begin{array}{l}4 \cdot 8 \\
0 \cdot 026\end{array}$ & $\begin{array}{l}8 \cdot 4 \\
0 \cdot 299\end{array}$ & $10 \cdot 6$ & $\begin{array}{l}6.8 \\
0.025\end{array}$ & $\begin{array}{l}10 \cdot 5 \\
0.275\end{array}$ \\
\hline$\Delta x$ & 0.063 & & & 0.075 & & & 0.048 & & \\
\hline $1 / 70$ & 0.9 & $2 \cdot 6$ & 3.9 & $2 \cdot 2$ & $2 \cdot 3$ & 3.7 & $5 \cdot 6$ & $4 \cdot 0$ & 5.7 \\
\hline${ }_{\Delta x}^{\Delta y}$ & 0.063 & 0.041 & 0.314 & 0.075 & 0.026 & $0-299$ & 0.048 & 0.025 & 0.275 \\
\hline
\end{tabular}

$d=1 / 70$, because transient simulations at high Rayleigh number are highly CPU-time consuming when using a commercial finite-element package. According to the finite element grid used, the Rayleigh number at which instabilities occur was found to be very close to $\mathrm{Ra}=7 \times 10^{7}$ and the system is then attracted to a limit cycle representing a periodic motion of frequency $f=357 \pm 38$. This Rayleigh number and frequency are close to those obtained with the source term formulation.

\subsection{Route to chaos of a buoyant plume in rectangular vessels}

On account of the rather good agreement between the solutions computed at high Rayleigh numbers by using one or the other of the two approaches, all the results presented in the following were obtained through the source term formulation.

4.2a Base flow simulations: Typical results for the streamlines and isotherms are shown in figures $8 \mathrm{a}-\mathrm{c}$ for various depths of immersion of line sources in the central vertical plane of the vessels. Maxima of the stream function are also given. All the simulations have been performed for air-filled vessels $(\operatorname{Pr}=0.71)$.

Table 8 gives the lower and upper bounds on the critical Rayleigh numbers. These bounds are for the highest value at which a steady-state motion was found to exist $\left(R a_{1}\right)$ and the smallest value at which the flow was found unsteady $\left(R a_{2}\right)$. The frequencies reported in table 8 are for the $\mathrm{Ra}_{2}$-values. The different types of bifurcation occurring in the configurations considered are also given. Steady-state isotherms and streamlines in square and rectangular vessels for Rayleigh numbers just below the first bifurcation point are shown in figure 8 . It can be seen that a recirculation flow is induced in which hot fluid rises with the plume above the line source, is cooled downstream and then descends along both sides of the vessel. The flow fields are 
(a)

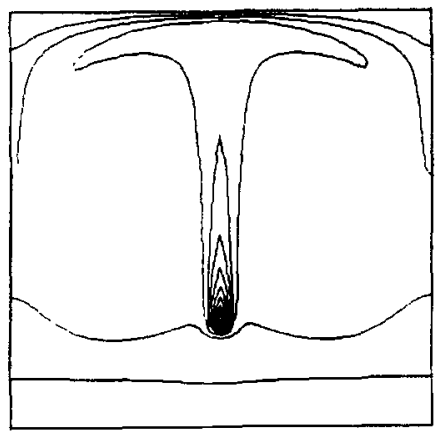

(b)

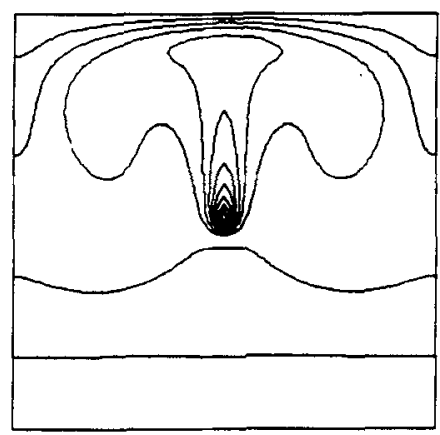

(c)

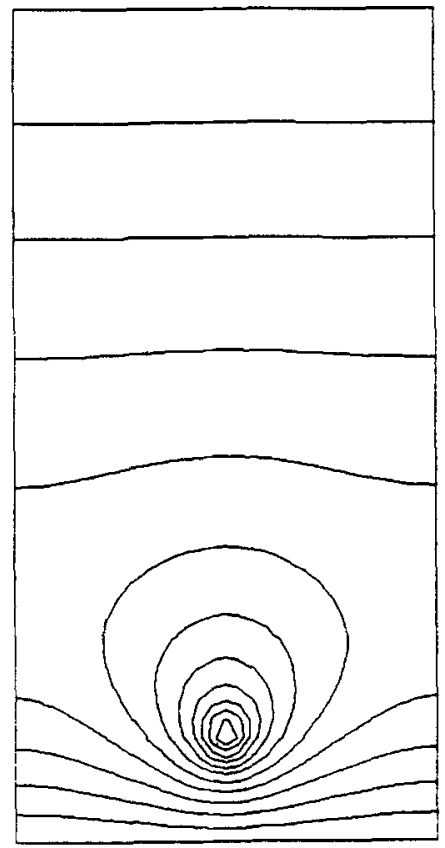

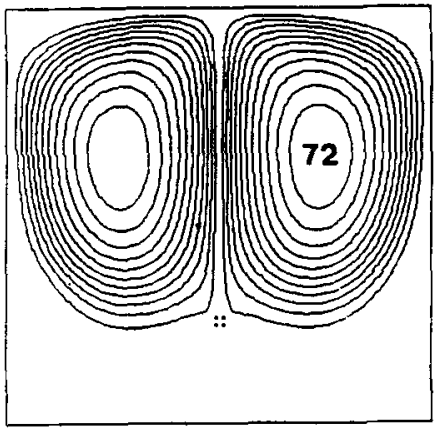
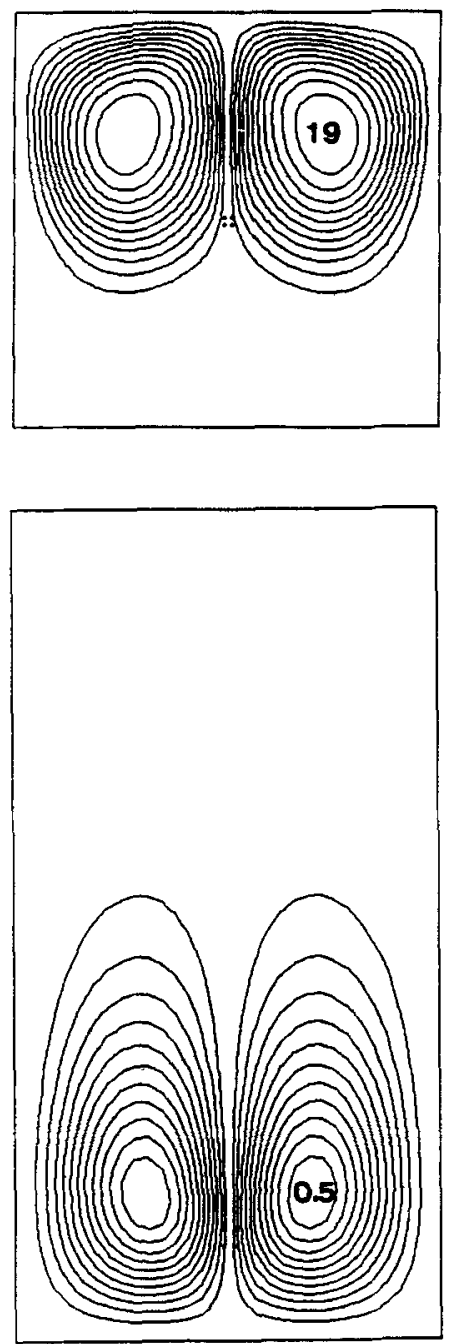

Figure 8. Steady-state isotherms (left) and streamlines (right) for various flows and Rayleigh numbers just below the critical values, $\operatorname{Pr}=0.71$. (a) $\operatorname{Ra}=3 \times 10^{7}$, $A=1, H_{\mathrm{s}}=0.75$; (b) $\mathrm{Ra}=1.9 \times 10^{6}, A=1, H_{\mathrm{s}}=0.50$; (c) $\mathrm{Ra}=5 \times 10^{3}, A=2$, $H_{\mathrm{s}}=1 \cdot 75$. The values given refer to stream function maxima. 
Table 8. Critical Rayleigh numbers, frequencies and types of Hopf bifurcation at various depths of immersion for $A=1$ (a) and 2 (b) and for various aspect ratios at $H_{\mathrm{s}}=0.75$ (c).

(a) $A=1$

\begin{tabular}{lcrc}
\hline$H_{s}$ & Bounds $\mathrm{Ra}_{1} \& \mathrm{Ra}_{2}$ & \multicolumn{1}{c}{$f$} & Type of Hopf bifurcation \\
\hline 0.75 & $3.0-3.1 \times 10^{7}$ & 294.2 & Supercritical \\
0.50 & $1.5-1.6 \times 10^{6}$ & 30.4 & Supercritical \\
0.25 & $3.1-3.2 \times 10^{6}$ & 38.5 & Supercritical \\
\hline
\end{tabular}

(b) $A=2$

\begin{tabular}{lcrl}
\hline$H_{\mathrm{s}}$ & Bounds $\mathrm{Ra}_{1} \& \mathrm{Ra}_{2}$ & \multicolumn{1}{c}{$f$} & Type of Hopf bifurcation \\
\hline 1.75 & $3.00-3.02 \times 10^{4}$ & 0.2 & Subcritical \\
1.50 & $2.1-2.2 \times 10^{4}$ & 1.7 & Subcritical \\
1.25 & $1.0-1.5 \times 10^{4}$ & 1.9 & Subcritical \\
1.00 & $2.0-2.5 \times 10^{4}$ & 5.3 & Supercritical \\
0.75 & $2.5-3.0 \times 10^{5}$ & 15.0 & Supercritical \\
\hline
\end{tabular}

(c) $H_{s}=0.75$

\begin{tabular}{lcrl}
\hline$A$ & Bounds $\mathrm{Ra}_{1} \& \mathrm{Ra}_{2}$ & $f$ & Type of Hopf bifurcation \\
\hline 2.00 & $2 \cdot 5-3.0 \times 10^{5}$ & 15.0 & Supercritical \\
1.75 & $3.0-4.0 \times 10^{5}$ & 17.2 & Supercritical \\
1.50 & $8 \cdot 0-9.0 \times 10^{5}$ & 25.6 & Supercritical \\
1.25 & $1.0-1.5 \times 10^{6}$ & 33.2 & Supercritical \\
1.00 & $3.0-3.1 \times 10^{7}$ & 294.2 & Supercritical \\
\hline
\end{tabular}

characterized by mirror symmetry about the vertical centreline. It is seen from figure $8 \mathrm{a}\left(A=1, H_{s}=0.75\right)$ that for line sources near the bottom wall, a strong steady fluid circulation occurs above the line source while a relatively stagnant layer of fluid is seen below. Obviously, the temperature gradients inside the bottom region strongly depend on the thermal boundary conditions applied at the bottom wall while the flow and temperature fields in the upper region of the vessel are weakly affected by this thermal boundary condition provided the Rayleigh number is large enough (Lauriat \& Desrayaud 1990). Similar features are seen in figure $8 \mathrm{~b}$ for smaller depth of immersion, $H_{s}=0.5$, but the bottom stagnant layer of fluid which is stably stratified now extends over one-third of the vessel approximately. On the other hand, for large depths of immersion in rectangular vessels as shown in figure $8 \mathrm{c}$, the plume does not reach the top wall and there is an unstably stratified layer of stagnant fluid above the plume. It should be noted that the fluid circulation is very weak owing to the low Rayleigh number. The flow is in a conductive state, as confirmed by the quasicircular isotherms around the source.

These three flow patterns give way to three different routes to chaos (Desrayaud \& Lauriat 1993). The first scenario, studied in the next sub-section, can be found only if the layer of fluid below the line source is small enough and if the plume reaches the top of the vessel. This happens only in vessels of small aspect ratio $(A \leqslant 1)$. The resulting periodic motion can be viewed as the natural swaying motion of confined plumes in the sense that the instabilities are neither driven by a stable layer of fluid 
at rest below the source as in figure $8 \mathrm{~b}$ nor triggered by an unstable layer of fluid above the plume as in figure $8 \mathrm{c}$. The two other scenarios are called penetrative convection, since the convecting plume is bounded below by a conducting layer of fluid, and Rayleigh-Bénard-like convection, since the underlying mechanism of the onset of instabilities is the destabilization of a motionless upper layer. These two mechanisms are respectively studied in $\S \S 4.2 \mathrm{c}$ and $4.2 \mathrm{~d}$ below.

Thus, contrary to what has been found in many studies on freely-rising thermal plume, interactions of the plume with the top and side walls play a dominant role in the flow structure.

4.2b Natural swaying motion in a square vessel: In this sub-section the numerical results discussed are for vessels of square cross-section, the line source being near the cold bottom wall $\left(H_{s}=0.75\right)$ as in figure $8 \mathrm{a}$.

Periodic motion-For Rayleigh numbers lower than $\mathrm{Ra}_{c}=3 \times 10^{7}$, the system is attracted to a fixed point, representing steady motion. The bifurcation leads to a system which is then attracted to a limit cycle indicating periodic motion.

The onset of unsteady solutions is due to the presence of a supercritical Hopf bifurcation point. Indeed, for such a bifurcation, the amplitude of the perturbation for slightly supercritical Rayleigh numbers evolves like $\left(\operatorname{Ra}-\mathrm{Ra}_{c}\right)^{0.5}$. This feature has been used to accurately determine the value of the critical point, which has been done from linear extrapolation of zero oscillation amplitude occurring close to the presumed threshold value. This gives a critical Rayleigh number very close to $3 \times 10^{7}$. A further feature of a Hopf bifurcation is the existence of a relationship between the dimensionless period of the oscillations and the Rayleigh number in the vicinity of the bifurcation point. By calculating the angular Brunt-Vaïsälä frequency associated with the plume for high Rayleigh numbers, it has been demonstrated that the frequency must be proportional to ( $\mathrm{Ra} \mathrm{Pr})^{0 \cdot 5}$ and should be nearly constant near the threshold (Desrayaud \& Lauriat 1993). This is well supported by the results of table 9 which shows the route to chaos, i.e. the nature of the bifurcation points and the associated frequencies.

A second frequency $f_{2}$ (given in parenthesis in table 9) appears at $\mathrm{Ra}=3.4 \times 10^{7}$ during the transient evolution but vanishes for a time unit greater than one, meaning that the frequency $f_{2}$ has at least eight orders of magnitude less power; moreover, these two frequencies are incommensurate.

During one period, the symmetry with respect to the vertical centre plane is respected. This finding was experimentally recorded by Yosinobu et al (1979) in the case of a buoyant plume in air. The general pattern of the mean temperature and stream function fields are the same as those presented in figure $8 \mathrm{a}$, but with higher isovalues. It can be noted that the upper part of the plume has the same symmetrical motion, once to the left, once to the right of the cavity. Hot and cold fluctuations grow simultaneously on each side of the source and a circulation of alternately hot and cold fluctuations arises in the two halves of the vessel. The instabilities are first confined within the plume where they are amplified and within the horizontal boundary layer near the top wall, then they move downward and back to the heat source. Therefore, instead of a swaying motion with sinusoidal wavelength as for a freely rising plume, we observed two counter-rotating circulations of fluid with alternating hot and cold spots. The motion of the plume itself is rather weak and detached blobs arise in the upper horizontal extents of the plume and sink along the 
Table 9. Route to chaotic motion in a square vessel with a line source at $H_{s}=0.75$

\begin{tabular}{|c|c|c|c|}
\hline $10^{-7} \mathrm{Ra}$ & Description & Frequency & $(\mathrm{Ra} / f)^{1 / 2}$ \\
\hline $\begin{array}{l}3 \cdot 00 \\
3 \cdot 10 \\
3 \cdot 20 \\
3 \cdot 30\end{array}$ & $\begin{array}{l}\text { Supercritical Hopf bifurcation } \\
\text { P1 } \\
\text { P1 } \\
\text { P1 }\end{array}$ & $\begin{array}{l}f_{1}=294 \cdot 2 \\
f_{1}=299 \cdot 7 \\
f_{1}=305 \cdot 2\end{array}$ & $\begin{array}{l}18 \cdot 93 \\
18 \cdot 87 \\
18 \cdot 82\end{array}$ \\
\hline 3.40 & Transient $\mathrm{QP} 2$, then $\mathrm{P} 1$ & $\begin{array}{c}f_{1}=310 \cdot 1 \\
\left(f_{2}=94 \cdot 4\right)\end{array}$ & $18 \cdot 80$ \\
\hline $3 \cdot 50$ & Transient QP2, then $\mathrm{P} 1$ & $\left\{\begin{array}{l}f_{1}=314 \cdot 8 \\
\left(f_{2}=96 \cdot 1\right)\end{array}\right.$ & 18.79 \\
\hline 3.60 & Transient QP2, then $P 1$ & $\left\{\begin{array}{l}f_{1}=319 \cdot 4 \\
\left(f_{2}=97 \cdot 7\right)\end{array}\right.$ & \\
\hline $3 \cdot 70$ & P2T (weak $f_{0}$ ) & $\left\{\begin{array}{c}f_{1}=323 \cdot 5 \\
\left(f_{2}=99 \cdot 2\right)\end{array}\right.$ & \\
\hline 3.80 & P2T (weak $f_{0}$ ) & $\left\{\begin{array}{l}f_{1}=328 \cdot 2 \\
f_{2}=101 \cdot 1\end{array}\right.$ & \\
\hline $\begin{array}{l}3.85 \\
3.90 \\
4 \cdot 00\end{array}$ & $\begin{array}{l}\text { Transient P2T, then I } \\
\text { Transient P2T, then I } \\
\text { I }\end{array}$ & & \\
\hline
\end{tabular}

P1 periodic state; QP2 quasi-periodic state with 2 incommensurate frequencies; P2T periodic state on a 2-torus; I intermittent state.

vertical adiabatic surface. Urakawa et al (1983) experimentally found identical behaviour in spindle oil but with a much stronger motion of the plume, especially just above the line source.

Two-frequency locked state - For $3.7 \times 10^{7} \leqslant \mathrm{Ra} \leqslant 3.8 \times 10^{7}$, the motion smoothly becomes a periodic, two-frequency locked state involving the $f_{1}$ and $f_{2}$ frequencies. The asymptotic state is then a limit cycle on a 2-torus of small cross-section. The phase portrait reveals that the trajectories are confined to a finite number of threads (figure 9a). The Poincaré section confirms this behaviour since 13 distinct group points are alternately visited in turn: for 13 rotations about its larger dimension, the trajectories pass four times around the smaller dimension (figure $9 \mathrm{~b}$ ). Thus, the rotation number (or the frequency-locking ratio) is $r=4 / 13$ and the fundamental frequency equals $f_{0}=f_{1}, 13=f_{2} / 4$. This is well supported by the frequency values reported in table 9 . Indeed, $f_{1} / f_{2}=3 \cdot 26 \pm 0.03 \cong 13 / 4$. Moreover, the $f_{2}$-frequency has only a weak influence on the whole motion, confined in the centre of the cells. It should also be noticed that the nonlinearities are weak since the contribution of the loworder mixing components $\left(f_{1}, f_{2}\right)$ is small. Simulations have been carried out up to 150,000 time-steps $(t>3)$, and no established quasi-periodic motion $\left(f_{1}, f_{2}\right.$ incommensurate) has ever been found.

Chaos - An intermittently chaotic state arises from the previous frequency-locked state. At irregular times and for irregular durations, the periodic laminar motion is interrupted by non-periodic 'bursts'. However, the characteristic of the frequencylocked state with locking ratio $4 / 13$ is maintained in the laminar windows. As a result, 


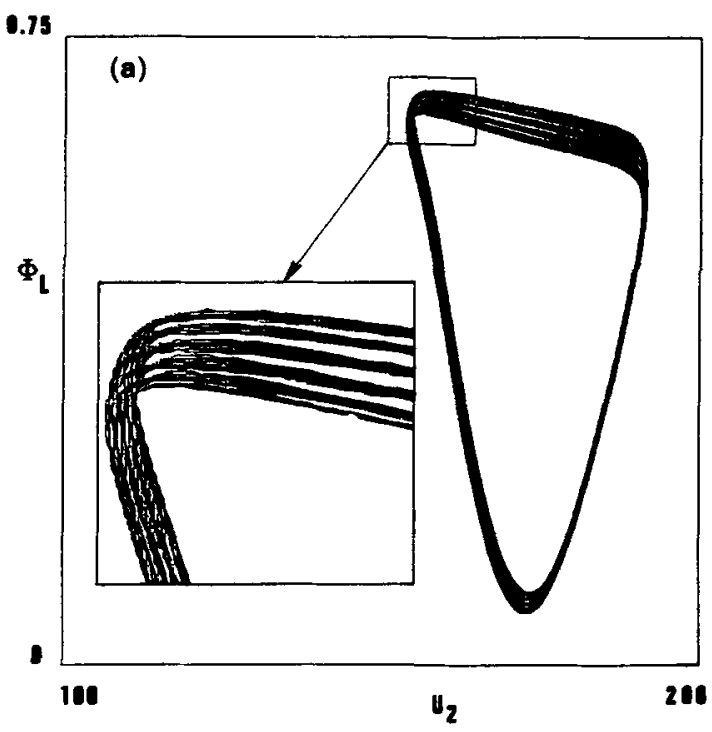

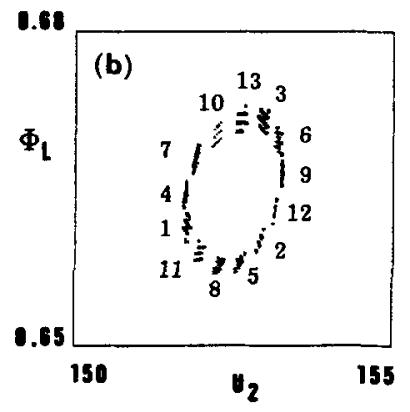

Figure 9. Phase portrait (a) and Poincaré map at surface-of-section $\phi_{r}=0.15$ (b) during 35,000 iterations or 0.525 time units, $A=1, H_{s}=0.75$, $\mathbf{R a}=3.85 \times 10^{7}$.

the spectrum exhibits broadband noise although relatively sharp spectral peaks still exist for all frequency multiples of the $4 / 13$ locked state (Lauriat \& Desrayaud 1990).

From their experiments, Forstrom \& Sparrow (1967) reported turbulent bursts at the beginning of the transition between the laminar and turbulent states (Note that Yosinobu et al (1979) did not observe such phenomena). In the transition regime, Bill \& Gebhart (1974) and Noto et al (1982) recorded transits from a turbulent state back to a laminar one, which seems like an intermittency phenomenon.

It could be concluded that a chaotic motion arises through a type-I intermittent transition. This type of intermittency is characterized by bursts of equal magnitude, periodic windows of identical frequencies, and near the transition, the lengths of these windows vary in proportion to $\left(\mathrm{Ra}-\mathrm{Ra}_{\mathrm{c}}\right)^{-0.5}$. The sequence of instabilities leading to non-periodic flows is also described on table 9 . The periodic two-frequency locked state has been abbreviated at P2T (periodic motion on a 2-torus).

4.2c Penetrative convection: As can be seen in figure $8 \mathrm{~b}$, there is a stable layer of fluid at rest at the bottom of the vessel for small depths of immersion although the convective motion extends slightly below the line source. Computations have been carried out in square and in rectangular vessels. For a square vessel, penetrative convection occurs if the depth of immersion is such that $H_{s} \leqslant 0.5$ while for rectangular vessels it happens when the depth of immersion is smaller than the width of the cavity (i.e., $H_{s} \leqslant 1$ ) (Desrayaud \& Lauriat 1993).

The bifurcation points are supercritical Hopf points with low frequency (cf table 8a, $H_{s} \leqslant 0.5$ and table $8 \mathrm{c}, A \geqslant 1.25$ ) since the amplitude increases roughly as the square root of the distance to the bifurcation point and the modified period is almost constant. The low values of the frequencies found can be explained by the fact that the plume has to set in motion the fluid below the source. This is illustrated in 


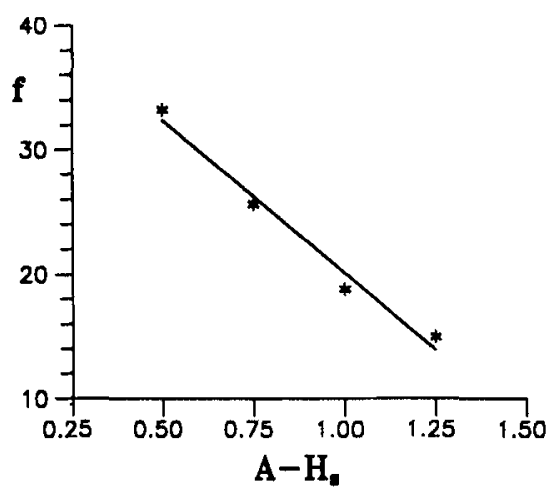

Figure 10. Variation of the frequency at critical points versus the height of the layer below the source for various aspect ratios and a fixed depth of immersion $H_{\mathrm{a}}=0.75$.

figure 10 which shows a linear variation of the frequency of the critical points (given in table $8 \mathrm{c})$ with the height of the layer below the source $\left(A-H_{s}\right)$ for a constant depth of immersion, $H_{s}=0.75$. Such a result clearly demonstrates that penetrative convection is the main phenomenon driving these instabilities. Some other cases in rectangular vessels and for depths of immersion $H_{s} \leqslant 1$ were investigated and the same trends were found.

In a square vessel and for a depth of immersion $H_{s}=0.75$, a periodic motion arises at $\mathrm{Ra}_{\mathrm{c}}=6 \times 10^{6}$ and is characterized by a large contribution from the two first harmonics. The flow then undergoes a second bifurcation into a limit cycle on a 2-torus. A weak frequency $f_{2}$, ten times smaller than the fundamental one $f_{1}$, appears at $\mathrm{Ra}=1.31 \times 10^{7}$ and a two-frequency locked state motion with a rotation number $r=f_{2} / f_{1}=1 / 10$ is obtained. The spectra of the trajectories exhibit a large number of sidebands around the fundamental peak and its harmonics resulting from a strong nonlinear interaction between the two-locked frequencies, $f_{1}$ and $f_{2}$. As the Rayleigh number is increased, these sidebands develop further, both in number and amplitude and compete with one another leading to the thickening of the torus. Just after the onset of chaotic behaviour the sidebands grow throughout the spectrum, the underlying envelop being broadband, despite the sharpness of the fundamental frequency $f_{1}$ and its two first harmonics. Thus, this results in a fast transition to a fully chaotic spectrum.

4.2d Rayleigh-Bénard-like convection: For rectangular vessels and depths of immersion greater than the width of the vessel $\left(H_{s}>1\right)$, very different bifurcations occur.

Pitchfork bifurcation - On increasing the Rayleigh number up to $\mathrm{Ra}=6000$, the symmetric two-cell pattern shown in figure $8 \mathrm{c}$ evolves towards an asymmetric one-cell pattern as in figure 11. The plume is deflected towards one vertical adiabatic wall, either left or right depending on the round-off errors generated during the computations. These two steady-state mirror image solutions characterize a pitchfork bifurcation (Desrayaud \& Lauriat 1993).

Contrary to what has been found for free laminar plumes, the destabilization of the two-cell flow comes from the unstably stratified layer of fluid at rest above the plume which appears when the plume does not reach the top of the vessel. Thus, Rayleigh-Bénard-like convection can arise in this upper layer when the Rayleigh 

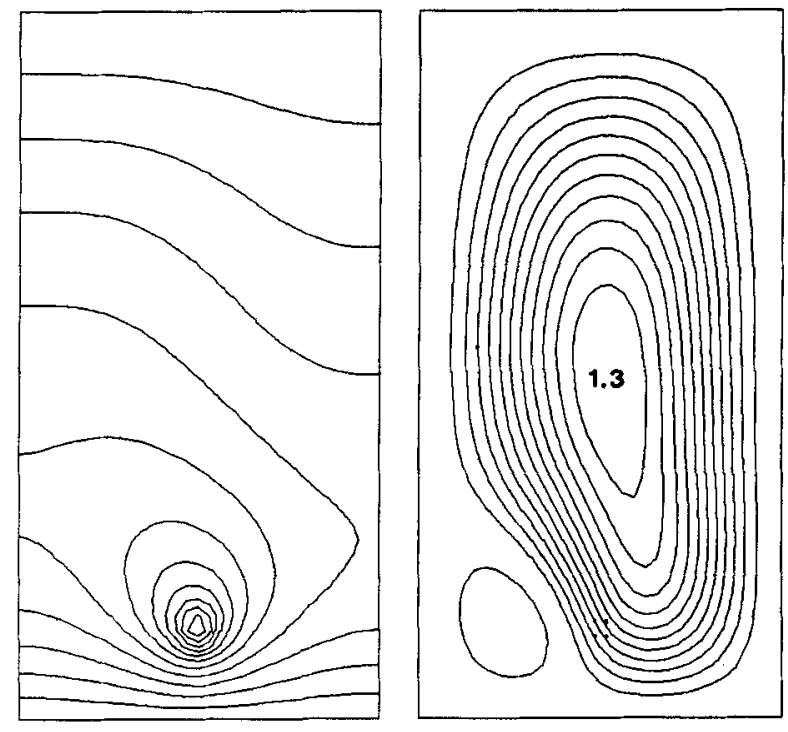

Figure 11. Isotherms and streamlines for stationary asymmetric flow, $A=2, H_{s}=$ $1 \cdot 75, \mathrm{Ra}=6 \times 10^{3}$. The values given refer to stream function maxima.

number is high enough, giving way to one-cell flow which spreads out in the vessel. The potential for multiplicities of steady-state mirror solutions is the result of nonlinearities of the governing equations. Similar behaviour has been observed by Hasnaoui et al (1990) for natural convection above an array of open cavities heated from below when the height of the vertical adiabatic confining walls is high enough.

Subcritical Hopf bifurcation - As the Rayleigh number is increased further, a sustained oscillatory convection is obtained through a subcritical Hopf bifurcation. Convenient variables to describe the temporal evolution of the flow are local variables on the centreplane of the vessel. Figure $12 \mathrm{a}$ presents the evolution of the horizontal velocity component $U$ at point $M_{1}(1 / 2,2 A / 3)$ for various Rayleigh numbers and for a depth of immersion $H_{s}=1 \cdot 75$. Below the pitchfork bifurcation point, which occurs at $\mathrm{Ra}=6 \times 10^{3}$, its value is zero due to the flow symmetry. Above it, the velocity component can take two opposite values. For higher Rayleigh numbers, the motion undergoes a second bifurcation at $\mathrm{Ra} \cong 3.02 \times 10^{4}$ into a limit cycle and the value oscillates periodically between the two mirror values of the Pitchfork bifurcation. Thus, oscillation onset is with finite amplitude which defines a subcritical Hopf bifurcation. On decreasing the Rayleigh number from $\mathrm{Ra}=3.02 \times 10^{4}$ to $3.01 \times 10^{4}$ the flow became steady again. Consequently, no hysteresis effect was found. As can be seen in figure $12 \mathrm{a}$, the value of the horizontal component of the velocity jumps at the Hopf bifurcation point. The underlying phenomenon is clearly depicted in figure $12 \mathrm{~b}$ which presents the time history of this variable for a Rayleigh number just above the onset of oscillations. Plateaus of opposite values which are those of the pitchfork bifurcation are periodically reached. The plume sways abruptly, briefly overshoots the other mirror flow solution due to its own inertia and becomes stable over a long period of time. At $\mathrm{Ra}=3.02 \times 10^{4}$ the period is equal to about 5 $(f \cong 0.2)$. This is why the frequencies are so small for $H_{s}>1$ (table $8 \mathrm{~b}$ ). On increasing 
(a) $\mathrm{U}$

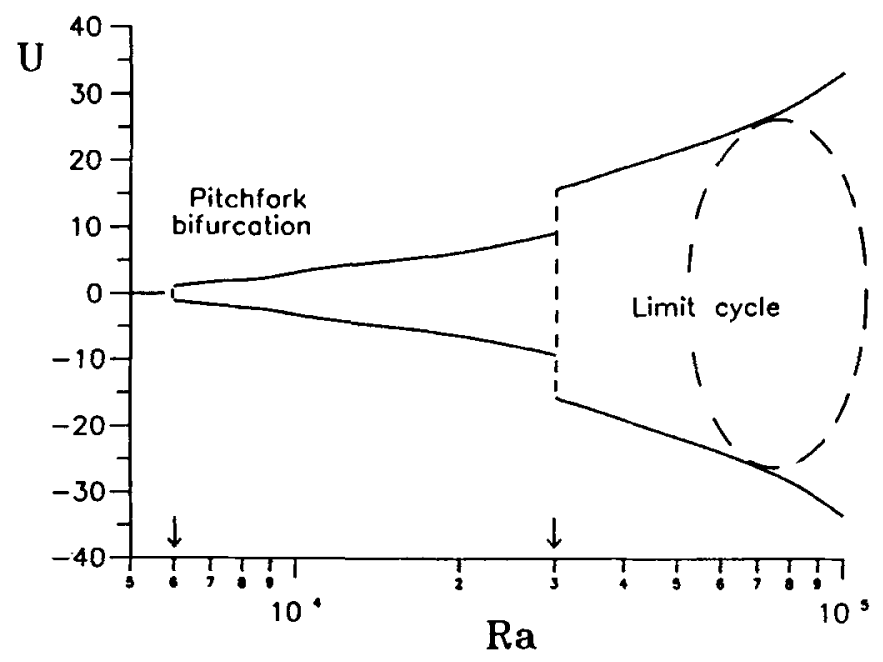

(b)

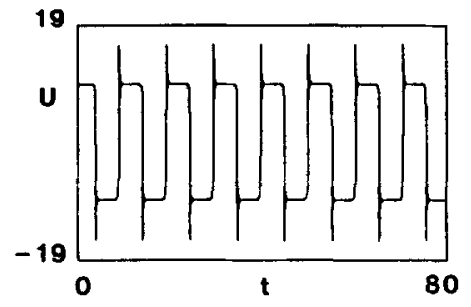

Figure 12. Evolution of the amplitude of the horizontal component of the velocity $U$ at point $(1 / 2,2 A / 3), A=2, H_{\mathrm{s}}=1 \cdot 75$ : (a) versus Rayleigh numbers; (b) at $\mathrm{Ra}=3.02 \times 10^{4}$.

the Rayleigh number, the solution continues to oscillate between two mirror solutions and the frequency is increased, the plateaus being shorter.

At higher $\mathrm{Ra}$, it seems that the temporal behaviour of the oscillations becomes chaotic. The leading Lyapounov exponent (LLE) estimate, which measures how unstable a given flow history is, has been calculated (Wolf et al 1985). Its sign provides a qualitative picture of the system's dynamics. The positive sign of the LLE implies the existence of chaotic behaviour at least for $\mathrm{Ra}=8 \times 10^{5}$. However, the regime is difficult to map accurately owing to limitations in the numerical resolution. Indeed, due to the very low value of the frequency, long time integrations are needed to correctly observe transitions and to obtain accurate power spectra. Nevertheless, it does not seem that any period doubling scenario exists after the pitchfork bifurcation as for the classical Feigenbaum scenario.

4.2e Comparisons with experimental results: Igarashi (1978) experimentally found that the swaying frequency is proportional to the 0.4 power of Rayleigh number for heated wires placed concentrically along the axes of horizontal rectangular chambers and thermal plumes reaching the ceiling of the vessel in a laminar state. Frequency correlations for various aspect ratios are depicted in figure 13. Three distinct groups of frequencies have been found as predicted experimentally by Igarashi (1978), one for $A \geqslant 1.8$, another for $0.7 \leqslant A \leqslant 1.5$ and the last for $A \leqslant 0.6$. Owing to the very high 


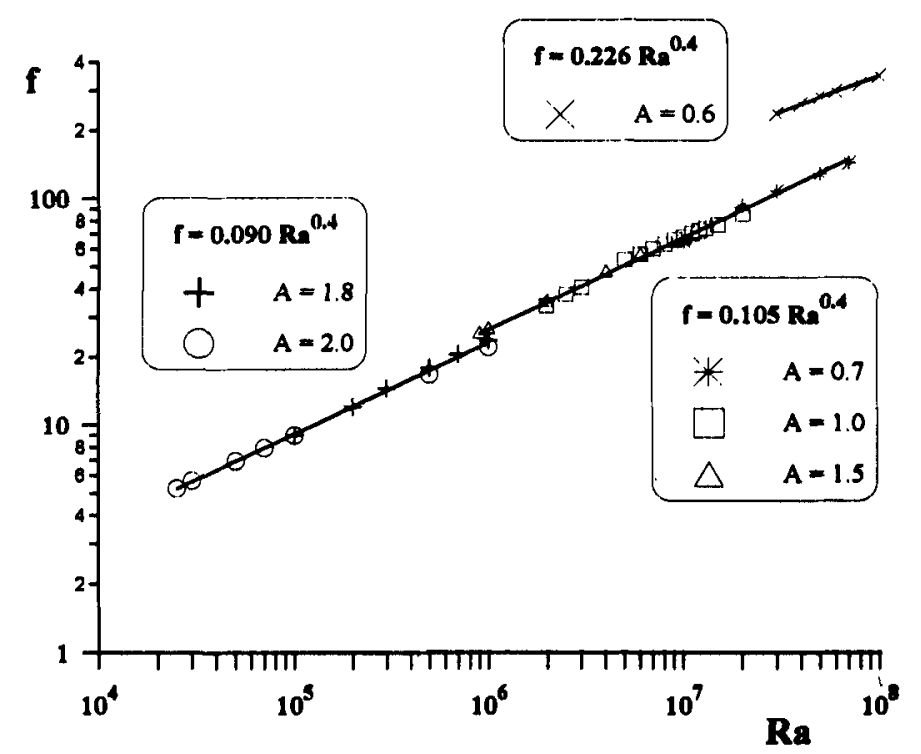

Figure 13. Evolution of the frequency for various aspect ratios and line heat source located at cavity mid-point.

Rayleigh number at which periodic motions appear for $A \leqslant 0.6$, computations have been made only for one aspect ratio, i.e. $A=0.6$. For each numerical frequency, the value of $f / \mathrm{Ra}^{0.4}$ has been calculated and the constants of the three correlations shown in figure 13 are the arithmetic mean values of each group of data. As can be seen, all the data are well correlated. The maximum discrepancies are lower than $5 \%$ except at $A=0.7$ where the differences reach $10 \%$.

The 0.4 power value has been recently confirmed by Noto \& Matsumoto (1986) and Noto (1989). The aspect ratio of their experimental set-up was $A=1.25$ with a dimensionless depth of immersion $H_{s}=0.75$. For the same geometrical parameters, a numerical frequency correlation has been computed, $f=0.109 \mathrm{Ra}^{0.4}$ with discrepancies lower than $3 \%$.

All these results give a good degree of confidence in the present results. It must be noted that the thermal boundary conditions play a minor role only. Indeed, in the experimental apparatus of Igarashi (1978) and Noto (1989), the temperatures of the ceiling, the bottom and the four sidewalls were isothermally controlled while our numerical boundary conditions are imposed by the top and bottom temperatures and vertical adiabatic walls.

Do the three correlations experimentally found by Igarashi (1978) correspond to the three oscillatory regimes numerically determined in the present study? To answer this question, all the numerical frequencies obtained in the present study are shown in figure 14, except those used to build the three correlations, i.e. for $H_{3}=A / 2$ (figure 13). This has been done to give a legible picture, and the correlations are reported in figure 14 as full lines. It is readily seen that the correlation $f=0.105 \mathrm{Ra}^{0.4}$ is for the penetrative convection regime; all the numerical frequencies corresponding to this regime agreed very well with this correlation. The other correlation, $f=$ 


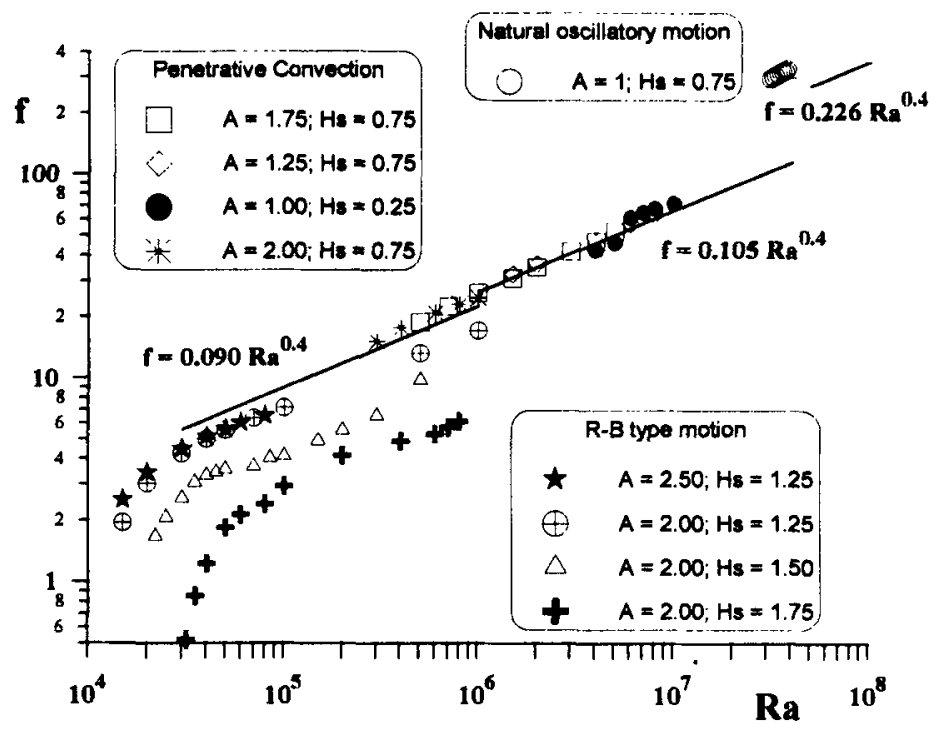

Figure 14. Comparison of the frequencies for various aspect ratios and depths of immersion given in figure 13.

$0.090 \mathrm{Ra}^{0.4}$, which is close to the previous one, also corresponds to the penetrative convection regime. In fact, it is a particular case of this regime for which there is competition between two modes, the Rayleigh-Bénard-like convection and the penetrative convection; for $H_{s} \cong 1$ and $A \cong 2$, as in the cases presented in figure 13 for this correlation, a pitchfork bifurcation arises first, due to the unstable layer of fluid at rest above the thermal plume. But this is followed by a resymmetrisation of the stationary regime, the mass of fluid below the source $\left(A-H_{s} \cong 1\right)$ being large enough to supply the fluid entrained downstream by the plume and restore the symmetry. For higher Rayleigh numbers, an oscillatory regime occurs through a supercritical Hopf bifurcation indicating that this regime can be classified as the penetrative convection regime. Such a sequence has been shown by Desrayaud \& Lauriat (1993, figure 10) for a rectangular vessel of aspect ratio 2. The third correlation, $f=0.226 \mathrm{Ra}^{0} 4$, seems to correspond to the natural swaying regime. At such high Rayleigh numbers very fine grids are needed and this can explain the large discrepancies found. Moreover, there are too few numerical points to extract some trends with confidence. Finally, owing to the geometrical parameters chosen by Igarashi $\left(H_{s}=A / 2\right.$ and $A \leqslant 2$ ), it was impossible to give prominence to the Rayleigh-Bénard-like regime which appears only when $H_{s}>1$. It is worth noting that this regime can be found in rectangular vessels of aspect ratio 2.5 (full stars in figure 14). For low and moderate Rayleigh numbers, the frequencies of the Rayleigh-Benard-like regime show that it does not follow the same type of correlation as the one found by Igarashi. The evolution of the frequencies for a given geometrical configuration does not follow a simple power-law, a sharp increase of the frequencies arising close to the bifurcation point when the Rayleigh number increases. For higher Rayleigh numbers, the evolution of the frequencies seems to be of the type determined by Igarashi, i.e. $\mathrm{Ra}^{0.4}$, but depends on the depth of immersion. 


\section{Conclusions}

The modelling of a heated wire as a cylinder of small diameter has been compared with a heat source formulation. For steady flows at low Rayleigh numbers, in the so-called conduction and transition regimes, fairly large discrepancies were found between the velocity fields because of the weak motion. However, the temperature field being unaffected by an obstacle, the agreement is much better for the heat transferred at the bottom and top cold surfaces. On the other hand, the velocity fields are in good agreement at high Rayleigh numbers, except just above the wire since the heat is then convected in a well defined plume. Only small differences in the isothermal patterns were found in the stagnant zone below the heat source. One of the most important parameters is the depth of immersion of the heat source. For unsteady regimes, both models show similar dynamical behaviour with very close frequencies for all of the types of bifurcation points investigated. Therefore, the heat source formulation was used to resolve the supercritical flows around a heated wire.

Swaying motions of confined thermal plumes above a horizontal line heat source inside a vessel with adiabatic side walls, cold top and bottom walls were investigated. The numerical predictions of the swaying frequency of laminar plumes were found in very good agreement with the experimental correlations of Igarashi (1978) and Noto (1989). A variety of dynamic behaviours were shown according to the depth of immersion, aspect ratio and Rayleigh number.

For rectangular vessels with $A>1$, two destabilizing mechanisms characterized by low frequency motions were found:

- for depths of immersion greater than the width of the vessel and small enough Rayleigh numbers, Rayleigh-Bénard-like instabilities may appear within the layer of fluid above the thermal plume. This results in asymmetric steady motions occurring through a pitchfork bifurcation, followed by a subcritical Hopf bifurcation.

- for depths of immersion smaller than the width, the mechanism driving the periodic motion is the penetrative convection which occurs within the layer of fluid at rest below the line heat source.

For square vessels, penetrative convection appears for rather small depths of immersion. Otherwise, for large depths of immersion, steady symmetric flows exist at high Rayleigh numbers. For $\mathrm{Ra}$ greater than a critical value which depends on the depth of immersion, a swaying motion with high frequency starts. This motion is followed by a two-frequency locked regime, then a weakly turbulent regime arises via an intermittent route to chaos.

\section{List of symbols}

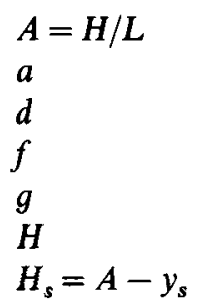

vertical aspect ratio;

thermal diffusivity;

dimensionless cylinder diameter;

frequency of time-dependent motion;

gravitational acceleration;

height of the vessel;

depth of immersion; 


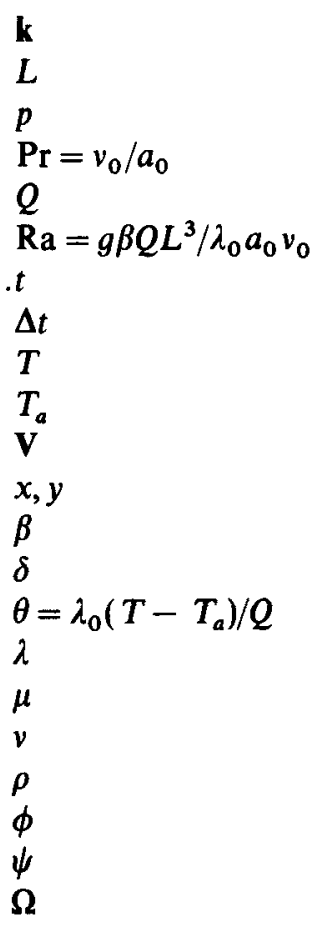

Subscripts

0

s

Superscripts unit vector in the $y$-direction;

width of the vessel;

pressure;

Prandtl number;

heat generated per unit length $(\mathrm{W} / \mathrm{m})$;

Rayleigh number;

time;

dimensionless time step;

temperature;

ambient temperature;

velocity vector of components $(U, V)$

coordinates;

volumetric thermal expansion coefficient;

Dirac delta function;

dimensionless temperature;

thermal conductivity;

fluid viscosity;

kinematic fluid viscosity;

fluid density;

dimensionless heat flux through a horizontal plane $y$;

dimensionless stream function;

dimensionless vorticity.

for reference temperature, refers to line source;

dimensional quantity.

\section{References}

Beck J V, McLain H A, Karnitz M A, Shonder J A, Segan E G 1988 Heat losses from underground steam pipes. J. Heat Transfer 110: 814-820

Bill R G, Gebhart B 1975 The transition of the plane plumes. Int. J. Heat Mass Transfer 18: 513-526

Brodowicz K, Kierkus W T 1966 Experimental investigation of laminar free-convection flow in air above horizontal wire with constant heat flux. Int. J. Heat Mass Transfer 9: 81-94

Crane L J 1959 Thermal convection from horizontal wire. Z. Angew. Math. Phys. 10:453-460

Deschamps V, Desrayaud G 1994 Modeling a horizontal heat-flux cylinder as a line source. AIAA J. Thermophys. Heat Transfer 8(1): 84-91

Deschamps V, Saiac J H 1992 Finite element simulation of thermal convection from horizontal cylinders embedded in boxes. Proc. 3rd European FID AP Users Group Meeting, Heidelberg, pp. $1-15$

Desrayaud G, Lauriat G 1991 On the difficulties in computing bifurcation points: application to buoyant plumes. High performance computing II (eds) M Durand, F El Dabaghi (Amsterdam: Elsevier Science, North-Holland)

Desrayaud G, Lauriat G 1993 Unsteady confined buoyant plumes. J. Fluid Mech. 252: 617-646

Desrayaud G, Lepeutrec Y, Lauriat G 1990 Numerical simulation of oscillatory convection in low-Pr fluids. Notes on numerical fluid mechanics (Braunschweig: Vieweg) 27: 49-56 
Eichhorn R, Lienhard J H, Chen C C 1974 Natural convection from isothermal spheres and cylinders immersed in a stratified fluid. Proc. 5th Int. Heat Transfer Conf., Tokyo 3: 10-14

Eichhorn R, Vedhanayagam M 1982 The swaying frequency of line source plumes. Proc. 7th Int. Heat Transfer Conf. Munich 2: 407-412

Engelman M S, Sani L, Gresho P M, Bercovier M 1982 Consistent versus reduced integration penalty methods for incompressible media using several old and new elements. Int. J. Numer. Methods Fluids 2: 25-42

Farouk B, Shayer H 1985 Natural convection around a heated cylinder buried in a saturated porous medium. Proc. 23th ASME Heat Transfer Conf. Denver 46: 181-189

FIDAP 1991 Theoretical manual, Vol. 1, Version 6.0 (Evanston, Il: Fluid Dynamics International)

Forstrom R J, Sparrow E M 1967 Experiments on the buoyant plumes above a heated horizontal wire. Int. J. Heat Mass Transfer 10: 321-331

Fujii T 1963 Theory of the steady laminar natural convection above a horizontal line heat source and a point heat source. Int. J. Heat Mass Transfer 6: 597-606

Fujii T, Fujii M, Honda T 1982 Theoretical and experimental studies of the free convection around a long horizontal thin wire in air. Proc. 7th Int. Heat Transfer Conf., Munich 2: 311-316

Fujii T, Morioka I, Uehara H 1973 Buoyant plume above horizontal line heat source. Int. J. Heat Mass Transfer 16: 755-768

Gebhart B, Jaluria Y, Mahajan R L, Sammakia B 1988 Buoyancy-induced flows and transport (New York: Hemisphere) chap. 3

Gebhart B, Pera L, Schorr A W 1970 Steady laminar natural convection plumes above a horizontal line heat source. Int. J. Heat Mass Transfer 13: 161-171

Golub G H, Meurant G A 1983 Résolution numérique des grands systèmes linéaires. Collection de la direction des Etudes et Recherches d'Electricité de France (ed) Eyrolles, (Paris:) chap. 3

Haaland S E, Sparrow E M 1973 Stability of buoyant boundary layers and plumes, taking into account nonparallelism of the basic flows. J. Heat Transfer 95C: 295-301

Hasnaoui M, Bilgen E, Vasseur P 1990 Natural convection above an array of open cavities heated from below. Numer. Heat Transfer 18A: 463-482

Hieber C A, Nash E J 1975 Natural convection above a line heat source: Higher-order effects and stability. Int. J. Heat Mass Transfer 18: 1473-1479

Himasekhar K, Bau H H 1988 Thermal convection around a heat source embedded in a box containing a saturated porous medium. J. Heat Transfer 110: 649-654

Huyakorn P S, Taylor C, Lee R L, Gresho P M 1978 A comparison of various mixedinterpolation finite elements in the velocity-pressure formulation of the Navier-Stokes equations. Comput. Fluids 6: 25-35

Igarashi T 1978 Natural convective oscillatory flow in an enclosed space. Part 2. Bull. Jpn. Soc. Mech. Eng. 21: 1022-1030

Igarashi T, Kada S 1977 Natural convective oscillatory flow in an enclosed space. Part 1. Heat Transfer Jpn. Res. 6(1): 19-40

Incropera F P, Yaghoubi M A 1980 Buoyancy driven flows originating from heated cylinders submerged in a finite water layer. Int. J. Heat Mass Transfer 23: 269-278

Jaluria Y 1982 Thermal plume interaction with vertical surfaces. Lett. Heat Mass Transfer 9: $107-117$

Kimura S, Bejan A 1983 Mechanism for transition to turbulence in buoyant plume flow. Int. J. Heat Mass Transfer 26: 1515-1532

Lauriat G, Desrayaud G 1990 Numerical study of oscillatory buoyant plumes above a horizontal line heat source. Proc. 9th Int. Heat Transfer Conf., Jerusalem 4: 171-176

Lin H T, Cheng W T 1992 Comprehensive correlations for laminar mixed convection line plume and wall plume. Int. J. Heat Mass Transfer 35: 2751-2753

Lyakhov Y N 1970 Experimental investigation of free convection above a heated horizontal wire. J. Appl. Mech. Tech. Phys. 11: 355-359

Maquet J, Gouesbet G, Berlemont A 1992 Numerical simulation of surface tension- and combined buoyancy-driven convection in a liquid layer heated by a hot wire. Int. J. Heat Mass Transfer 35: 2695-2703

Morwald K, Mitsotakis K, Schneider W 1986 Higher-order analysis of laminar plumes. Proc. 8th Int. Heat Transfer Conf., San Francisco 3: 1335-1340

Nawoj H J, Hickman R S 1977 An experimental investigation of the plume velocity field above a horizontal line heat source. J. Heat Transfer 99: 609-613 
Noto K 1989 Swaying motion in thermal plume above a horizontal line heat source. $J$. Thermophys. 3: 428-434

Noto K, Matsui S, Matsumoto R 1982 Observation on vortex pair of plane plume in thermally stratified fluid. Flow visualization (Berlin: Springer Verlag) vol. 4

Noto K, Matsumoto R 1986 Swaying motion in buoyant air plume above a horizontal line heat source. AIAA/ASME 4th Joint Thermophysics and Heat Transfer Conf., Boston, paper 86-1266, pp. $1-9$

Pera L, Gebhart B 1971 On the stability of laminar plumes: Some numerical solutions and experiments. Int. J. Heat Mass Transfer 14: 975-984

Pera L, Gebhart B 1975 Laminar plume interactions. J. Fluid Mech. 68: 259-271

Peyret R 1990 The Chebyshev multidomain approach to stiff problems in fluid mechanics. Methods Appl. Mech. Eng. 80: 129-145

Roache P 1982 Computational fluid dynamics (Albuquerque: Hermosa)

Roux B (ed.) 1990 Numerical simulation of oscillatory convection in low-Prandtl fluids. Notes on numerical fluid mechanics (Braunschweig: Vieweg) vol. 27

Reimann J 1974 Experimental investigation of free convection flow from wires in the vicinity of phase interfaces. Int. J. Heat Mass Transfer 17: 1051-1061

Roze C, Gouesbet G, Darrigo R 1993 Oscillatory instabilities produced by heat from a temperature-controlled hot wire below an interface. J. Fluid Mech. 250: 253-276

Schorr A W, Gebhart B 1970 An experimental investigation of natural convection wakes above a line heat source. Int. J. Heat Mass Transfer 13: 557-571

Sohn J L 1988 Evaluation of FIDAP on some classical laminar and turbulent benchmarks. Int. J. Numer. Methods Fluids 8: 1469-1490

Urakawa K, Morioka I, Kiyota M 1983 Swaying motion of the buoyant plume above a horizontal line heat source. Proc. 1st ASME-JSME Thermal Eng. Conf., Honolulu 3: 215-220

Wakitani S 1985 Non-parallel-flow stability of a two-dimensional buoyant plume. J. Fluid Mech 159: 241-258

Wakitani S, Yosinobu H 1984 Stability characteristics of a natural convection flow above a horizontal line heat source. J. Phys. Soc. Jpn. 53: 1291-1300

Wolf A, Swift J B, Swinney H L, Vastano J A 1985 Determining Lyapounov exponents from a time series. Physica D16: 285-317

Xia J L, Xin M D, Zhang H J 1990 Natural convection in an externally heated enclosure containing a local heat source. J. Thermophys. Heat Transfer 4: 233-238

Xia J L, Xin M D, Zhang H J 1991 A numerical study of natural convection in an externally heated enclosure with a local heat source. Proc. 9th Int. Heat Transfer Conf., Jerusalem 4: 217-222

Yaghoubi M A, Incropera F P 1978 Natural convection from a heated horizontal cylinder submerged in a shallow water layer. Proc. 6th Int. Heat Transfer Conf., Toronto 2: 269-274

Yang H Q 1992 Buckling of a thermal plume. Int. J. Heat Mass Transfer 35: 1527-1533

Yosinobu H, Onishi Y, Amano S, Enyo S, Wakitani S, 1979 Experimental study on instability of a natural convection flow above a horizontal line heat source. J. Phys. Soc. Jpn. 47: 312-319 